\title{
Quadratic voting with multiple alternatives
}

\author{
Jon X. Eguia* Nicole Immorlica $^{\dagger} \quad$ Katrina Ligett $^{\ddagger} \quad$ Glen Weyl ${ }^{\S}$ \\ Dimitrios Xefteris
}

April 3, 2019

\begin{abstract}
Consider the following collective choice problem: a society of budget-constrained agents faces multiple alternatives and wants to reach an efficient decision (i.e. to Nash implement the utilitarian maximum). In this paper, we propose a budget-balanced vote-buying mechanism for this setting: for each alternative, every voter can cast any number of votes, $x$, in support or against it, by transferring an amount $x^{2}$ to the rest of the voters; and the outcome is determined by the net vote totals. We prove that as the society grows large, in every equilibrium of the mechanism, each agent's transfer converges to zero, and the probability that the mechanism chooses the socially efficient outcome converges to one.
\end{abstract}

Keywords: implementation, efficiency, mechanism design, quadratic voting, multiple alternatives.

JEL classification: D72, D71, D61.

*Michigan State U. eguia@msu.edu

${ }^{\dagger}$ Microsoft

${ }^{\ddagger}$ Hebrew U.

$\S$ Microsoft and Princeton

ฯU. Cyprus. 
Consider a collective choice problem with many strategic agents and multiple alternatives. Assume the group facing this collective choice wishes to choose the alternative that maximizes the sum of utilities (the utilitarian optimum), and wants to make this choice through a procedure that is easy to execute. By "easy to execute" we mean a procedure that does not require a budget sacrifice to run (budget-balance), and that makes it possible for agents with time or budget constraints to participate, by not requiring agents to report complex messages and by minimizing monetary transfers across agents.

Even under a restriction of the preference domain to quasilinear profiles (preferences that are separable over the alternative and wealth, and risk neutral on wealth), any group facing a collective-choice with multiple alternatives encounters a difficult challenge: in order for the choice to always be the utilitarian optimum for any equilibrium for any publicly revealed realization of preferences, the decision-making mechanism must Nash implement the utilitarian choice rule. Unfortunately, mechanisms known to Nash implement the utilitarian rule (e.g. Maskin 1999), use very complex message spaces, and are thus hard to use in any environment of applied interest.

The VCG mechanisms of Vickrey (1961), Clarke (1971) and Groves (1973) require only simpler messages, of dimension equal to the number of alternatives, and they feature one equilibrium that chooses the optimal alternative. However, these mechanisms are not budgetbalanced, they require large transfers, and they also feature equilibria that do not choose the optimal alternative. We can overcome the first problem by running a VCG mechanism with $n-1$ agents, and letting the $n-t h$ agent be the residual claimant of all transfers; the resulting mechanism is budget balanced and features an equilibrium that is asymptotically optimal, in the sense that the probability that it chooses optimally converges to one in the size of the group (Jackson 2000). But this approximate-VCG mechanism also features asymptotically inefficient equilibria, and it still requires large transfers, so it cannot run if agents with intense preferences about the alternatives have tight individual budget constraints. ${ }^{1}$

We propose a mechanism that satisfies almost all the desiderata laid out in the first paragraph: the mechanism is asymptotically optimal for every equilibrium, so it asymptotically Nash implements the utilitarian choice rule; is budget balanced; uses a message space of dimensionality as small as that of the VCG mechanism (as many dimensions as the number of alternatives); and it requires only vanishing transfers as the group becomes arbitrarily large. In short, the mechanism is easy to run, and it converges toward utilitarian optimality

\footnotetext{
${ }^{1}$ For details on efficiency results with different notions of incentive compatibility, see the mechanism design survey by Börgers (2015). For Nash implementation, surveys by Jackson (2001) or Maskin and Sjöström (2002).
} 
for large groups of (potentially) budget-constrained agents. ${ }^{2}$

The mechanism we propose is a vote-buying mechanism with quadratic costs, in which agents can express any intensity of support or opposition to each alternative, at a cost that is the square of the intensity expressed. The total costs for an individual is aggregated across all alternatives, and this total cost is redistributed in the form of a transfer to all other agents. The choice is based on the net support for each alternative, so that the probability that an alternative is chosen increases in its net support.

This mechanism generalizes the "quadratic voting" mechanism for binary choices (Lalley and Weyl 2016, 2018) to an environment with multiple alternatives. ${ }^{3}$ Under the restrictive but standard assumptions that agents' preferences over the choice of alternative and over wealth are separable, and that agents are risk neutral, we show that the mechanism is asymptotically efficient: the mechanism chooses the alternative that maximizes social welfare with a probability that converges to one as the size of the group diverges to infinity. In fact, the mechanism completely orders alternatives by their utilitarian order: the utilitarian optimum earns most net support, the socially second-best alternative obtains the second highest net support, etc. We also find that individual equilibrium costs and transfers converge to zero, so that any non-zero individual budget constraint becomes non-binding.

Some alternative mechanisms, such as voting procedures that choose the alternative that gets most votes are easy to use and -under some conditions- are optimal for binary choices (Börgers 2004; Krishna and Morgan 2015), but they do not work well for choice among multiple alternatives: they have multiple equilibria, many of them inefficient. In some environments, voters may behave truthfully because devising manipulations is computationally infeasible (Conitzer et al. 2007; Faliszewski and Procaccia 2010; Brandt et al. 2016; Meir 2018), and best-response dynamics can lead to outcomes that are nearly optimal according to some some scoring rules (Branzei et al. 2013). However, these outcomes obtained by truthful strategy profiles, or by learning through best response dynamics, can also fail to attain utilitarian efficiency. In contrast, under our mechanism, society asymptotically attains efficiency in all equilibria.

\footnotetext{
${ }^{2}$ Our mechanism is "easy" to run in the sense that the message space required is small. Other approaches that constrain the set of mechanisms under consideration require the mechanisms to be "bounded" (Jackson 1992); or easy to play by being "strategically simple" (Börgers and Li 2018) or "obviously strategy-proof" (Li 2017).

${ }^{3}$ On quadratic and other vote-buying mechanisms for binary choices, see as well Goeree and Zhang (2017) and Eguia and Xefteris (2018). The suggestion that the votes cast by an entity should be the square root of its size dates back at least to Penrose (1946), and quadratic costs also arise as the limit of d'Aspremont and Gerard-Varet's (1979) mechanism as the size of the society diverges to infinity (Goeree and Zhang 2017).
} 
Given that our mechanism's efficiency result hinges on the agents being risk neutral, we refrain from proposing that democracies use it as the main means to aggregate (risk-averse) citizen's preferences over ideological choices. ${ }^{4}$ Rather, a more suitable application is collective choice by agents who maximize expected profits, such as any profit-maximizing firms. Applications of particular interest include governance in cryptocurrencies, and collective choice in chambers of commerce or businesses' associations.

\section{Model}

Alternatives, agents and values. Let $X \equiv\{1,2, \ldots, m\}$ be a finite set of alternatives, $m \in \mathbb{N}, m \geq 3 .^{5}$ Let $\mathbb{N}$ be a society with a countably infinite number of agents.

For each agent $i \in \mathbb{N}$ and each $k \in X$, let $u_{k}^{i} \in[0,1]$ denote the value that agent $i$ assigns to alternative $k$ (throughout the document, superscripts refer to agents, and subscripts to alternatives). This value can be understood as a quantity of real wealth that the agent would be willing to trade to obtain alternative $k$, instead of an outside worst alternative with value normalized to zero. Let $u^{i} \equiv\left(u_{1}^{i}, \ldots, u_{m}^{i}\right) \in[0,1]^{m}$ denote the vector of values for agent $i$. Assume that for each $i \in \mathbb{N}, u^{i}$ is independently drawn from a distribution $F$ over $[0,1]^{m}$, with expected value $\left(u_{1}, \ldots, u_{m}\right) \in(0,1)^{m}$, where for each $k \in X$, $u_{k}$ denotes the expected value of alternative $k$. Assume that these expected values are all distinct, and, without loss of generality, that alternatives are labeled from best to worst in expectation so that $u_{1}>u_{2}>\ldots>u_{m}$.

Let $[0,1]^{\mathbb{N} \times m}$ denote the set of all matrixes with countably infinitely many rows, $m$ columns, and cell values in $[0,1]$. Let $v \equiv\left(\begin{array}{c}u^{1} \\ u^{2} \\ \vdots\end{array}\right)=\left(\begin{array}{ccc}u_{1}^{1} & \ldots & u_{m}^{1} \\ u_{1}^{2} & \ldots & u_{m}^{2} \\ \vdots & \ldots & \vdots\end{array}\right) \in[0,1]^{\mathbb{N} \times m}$ denote the infinite matrix of value vectors, containing the value of each alternative for each agent $i \in \mathbb{N}$, and let $v^{n} \equiv\left(\begin{array}{c}u^{1} \\ \vdots \\ u^{n}\end{array}\right)=\left(\begin{array}{ccc}u_{1}^{1} & \ldots & u_{m}^{1} \\ \vdots & \ldots & \\ u_{1}^{n} & \ldots & u_{m}^{n}\end{array}\right) \in[0,1]^{n \times m}$, denote the first $n$ rows of matrix $v$.

The game with $n$ agents. For any finite size $n \in \mathbb{N} \backslash\{1\}$, we consider the choice problem of

\footnotetext{
${ }^{4} \mathrm{~A}$ possible alternative for these applications is storable votes (Casella 2005), or a combination of storable votes and quadratic voting, such that voters are endowed with a stock of rights they can store and they can convert these rights into votes at a quadratic cost on any given decision.

${ }^{5}$ Results apply as well for $m=2$ as a special case.
} 
choosing an alternative in $X$ faced by the finite subset $\{1,2, \ldots, n\} \in \mathbb{N}$ of the society, given the matrix of values $v^{n}$.

Assumption 1 For each $n \in \mathbb{N}$, matrix $v^{n}$ is common knowledge among the subset of agents $\{1,2, \ldots, n\}$.

Given the realization of the matrix of values $v$, and for each $n \in \mathbb{N} \backslash\{1\}$, society $N(n)$ solves its collective choice problem by means of a quadratic vote-buying mechanism. A quadratic vote-buying mechanism for a binary choice problem (Lalley and Weyl 2018), is such that each participant can cast any real-valued quantity of votes, and the alternative that the proposal passes is increasing in the net total quantity of votes. The mechanism charges each agent a monetary payment equal to the square of the votes cast by the agent, and redistributes the proceeds to all other agents, so that the mechanism is budget balanced.

We generalize this mechanism to multiple alternatives: each agent chooses how many votes to cast for or against each alternative, resulting in a net total for each alternative. The chosen alternative is a probabilistic function of net vote totals. For each alternative, each agent is charged the square of her votes for this alternative, so that an agent's total charge is the sum of these squares. Proceeds are redistributed to all other agents, to make the mechanism budget-balanced.

Note the mechanism's minimal requirements of information: in order to run, all that the mechanism (or the institution in charge of running it) needs is the means to verify whether any agent trying to submit an action is a member of the society. The mechanism does not need to know the number $n$ of agents in the society, nor the distribution $F$ from which values are drawn, not even the domain of this distribution.

Let $\Gamma\left(v^{n}\right)$ denote the game induced by this quadratic voting mechanism, for a given realization of the matrix of values $v$, and for a given $n \in \mathbb{N} \backslash\{1\}$.

The set of players in game $\Gamma\left(v^{n}\right)$ is the set of agents $\{1,2, \ldots, n\}$.

Agent $i$ as a player in game $\Gamma\left(v^{n}\right)$ chooses an action $a^{i}\left(v^{n}\right) \equiv\left(a_{1}^{i}\left(v^{n}\right), \ldots, a_{m}^{i}\left(v^{n}\right)\right) \in$ $[-1,1]^{m}{ }^{6}$ A pure strategy for agent $i$ in game $\Gamma\left(v^{n}\right)$ is just a choice of an action, so the set of pure strategies for each agent is $S=[-1,1]^{m}$, and the set of strategy profiles is $S^{n}=[-1,1]^{n \times m}$.

Each component of the vector $a^{i}\left(v^{n}\right)$ can be interpreted as a quantity of votes cast for or

\footnotetext{
${ }^{6}$ We can allow for unbounded action spaces, but we do not need them for our results.
} 
against each alternative, or it can more accurately be interpreted as an expression of intensity of support or opposition to each alternative. Let $a\left(v^{n}\right) \equiv\left(\begin{array}{c}a^{1}\left(v^{n}\right) \\ a^{2}\left(v^{n}\right) \\ \ldots \\ a^{n}\left(v^{n}\right)\end{array}\right)$ denote the matrix of actions taken by all agents in game $\Gamma\left(v^{n}\right)$, and let $a^{-i}\left(v^{n}\right)$ be the matrix without row $i$. For each alternative $k \in X$, define

$$
\begin{aligned}
A_{k}\left(v^{n}\right) & \equiv \sum_{j=1}^{n} a_{k}^{j}\left(v^{n}\right) \\
A_{k}^{-i}\left(v^{n}\right) & \equiv \sum_{j=1}^{n} a_{k}^{j}\left(v^{n}\right)-a_{k}^{i}\left(v^{n}\right), \text { and } \\
A\left(v^{n}\right) & \equiv\left(A_{1}\left(v^{n}\right), \ldots, A_{m}\left(v^{n}\right)\right) .
\end{aligned}
$$

Note $A_{k}\left(v^{n}\right) \in[-n, n], A_{k}^{-i}\left(v^{n}\right) \in[-n, n]$, and $A\left(v^{n}\right) \in[-n, n]^{m}$.

Given $a\left(v^{n}\right)$, the quadratic voting mechanism chooses alternative $k$ with probability

$$
p_{k}\left(A\left(v^{n}\right)\right) \equiv \frac{e^{A_{k}\left(v^{n}\right)}}{\sum_{h=1}^{m} e^{A_{h}\left(v^{n}\right)}}
$$

The substantively relevant property of this outcome function is that holding constant the net vote totals for other alternatives, the probability that alternative $k$ is chosen is strictly increasing in the net vote total for $k^{7}$

Each player $i$ incurs a cost of her action equal to $c \sum_{k=1}^{m}\left(a_{k}^{i}\left(v^{n}\right)\right)^{2}$, where $c \in \mathbb{R}_{++}, c>\frac{1}{2}$. These costs are interpreted as a monetary contribution to the mechanism, associated to any given action. The mechanism then redistributes all these contributions equally among all other agents, so as to be budget-balanced.

The utility for player $i$ as a function of $a\left(v^{n}\right)$ is

$$
U^{i}\left(a\left(v^{n}\right)\right) \equiv \sum_{k=1}^{m} p_{k}\left(A\left(v^{n}\right)\right) u_{k}^{i}-c \sum_{k=1}^{m}\left(a_{k}^{i}\left(v^{n}\right)\right)^{2}+\frac{c}{n-1} \sum_{j \in N(n) \backslash\{i\}} \sum_{k=1}^{m}\left(a_{k}^{j}\left(v^{n}\right)\right)^{2}
$$

\footnotetext{
${ }^{7}$ Our results hold if we assume instead that, for some $\phi \in \mathbb{R}_{++}, p_{k}\left(A\left(v^{n}\right)\right) \equiv \frac{e^{\phi A_{k}\left(v^{n}\right)}}{\sum_{h=1}^{m} e^{\phi A_{h}\left(v^{n}\right)}}$, in which case, as $\phi$ becomes large, the outcome function approximates a step function that selects the alternative with most net votes. For the sake of a more parsimonious exposition, we choose $\phi=1$.
} 
Let $U^{v, n}:[-1,1]^{n \times m} \longrightarrow \mathbb{R}^{n}$ be the function that maps an action (or strategy) profile into a utility profile, defined by

$$
U^{v, n}\left(a\left(v^{n}\right)\right)=\left(U ^ { 1 } \left(a\left(v^{n}\right), \ldots, U^{n}\left(a\left(v^{n}\right)\right) .\right.\right.
$$

Then $\Gamma\left(v^{n}\right) \equiv\left(\{1,2, \ldots\}, S^{n}, U^{v, n}\right)$ is the complete information, normal form game played by the subset of agents $\{1,2, \ldots, n\}$ given the realization of the matrix of values $v$.

A large number of agents. Given the realization of the matrix of values $v$, let $\left\{\Gamma\left(v^{n}\right)\right\}_{n=2}^{\infty}$ denote the sequence of games, one for each size $n \in \mathbb{N}$. Note that each agent $i \in \mathbb{N}$ plays in infinitely many separate games: $i$ is a player in each game in the subsequence $\left\{\Gamma\left(v^{n}\right)\right\}_{n=i}^{\infty}$. Each game is played separately, and while for each player $i$, the vector of values $u^{i}$ is constant across all games (it is the $i-t h$ row of matrix $v$ ), player $i$ can play a different action in each game $\left(a^{i}\left(v^{n}\right)\right.$ conditions on $v^{n}$, which varies across games).

We consider a sequence of pure Nash equilibria that corresponds to this sequence of games. We identify the asymptotic welfare properties of these equilibria as the number of agents participating in the collective decision becomes sufficiently large.

\section{Asymptotic optimality of quadratic voting}

We first prove a preliminary result: that if the cost parameter $c$ is sufficiently large, for each realization of the value matrix $v$, and for each $n \in \mathbb{N} \backslash\{1\}$, a pure Nash equilibrium of game $\Gamma\left(v^{n}\right)$ exists. $^{8}$

The intuition to establish this existence result is as follows. Fix $v \in[0,1]^{\mathbb{N} \times m}$ and $n \in$ $\mathbb{N} \backslash\{1\}$. The number of players in game $\Gamma\left(v^{n}\right)$ is finite: specifically, it is $n$. Their action set is bounded. Their utilities, given by Expression (2), are continuous in actions, and bounded as well. We establish that the utility function (2) is concave in $a^{i}\left(v^{n}\right)$, which then implies existence of an equilibrium. All proofs are in the Appendix.

Lemma 1 There exists $\bar{c} \in \mathbb{R}_{++}$such that, if $c>\bar{c}$, for any $v \in[0,1]^{\mathbb{N} \times m}$, and for any $n \in \mathbb{N} \backslash\{1\}$, a pure Nash equilibrium of game $\Gamma\left(v^{n}\right)$ exists.

Any equilibrium is interior: corner solutions do not exist. Let $a^{*}\left(v^{n}\right) \in[-1,1]^{n \times m}$ denote

\footnotetext{
${ }^{8}$ Numerical computations for $m=3$ show that a value of $c$ as low as $c=1$ suffices to obtain existence.
} 
an equilibrium of game $\Gamma\left(v^{n}\right)$, where $a_{k}^{*, i}\left(v^{n}\right) \in[-1,1]$ is the equilibrium action of agent $i$ on alternative $k$.

Lemma 2 For any $v \in[0,1]^{\mathbb{N} \times m}$, and for any $n \in \mathbb{N} \backslash\{1\}$, any equilibrium of game $\Gamma\left(v^{n}\right)$ is interior, so $a_{k}^{*, i}\left(v^{n}\right) \in(-1,1)$ for any agent $i$ and for any alternative $k$.

Having established that if $c>\bar{c}$, then a pure Nash equilibrium exists in the game with a finite subset of the society, for any size of this finite subset, and for any realization of values, we next derive asymptotic welfare properties of all such equilibria, if the subset of participating agents is sufficiently large.

We consider a sequence of games with increasing number of players, and all sequences of equilibria for these games. We obtain results for all equilibria sufficiently far along the sequence. For each $n \in \mathbb{N}$, and for each alternative $k \in X$, let $\bar{u}_{k}\left(v^{n}\right)$ denote the value that agents 1 through $n$ assign on average to alternative $k$, so

$$
\bar{u}_{k}\left(v^{n}\right) \equiv \frac{1}{n} \sum_{i=1}^{n} u_{k}^{i} .
$$

By the (strong) law of large numbers, this observed average value $\bar{u}_{k}\left(v^{n}\right)$ converges almost surely to the expected value $u_{k}$ :

$$
\operatorname{Pr}\left[\lim _{n \longrightarrow \infty} \bar{u}_{k}\left(v^{n}\right)=u_{k}\right]=1
$$

The probability is with respect to the realization of the matrix of values $v$. In particular, for $d=\frac{\min _{k \in\{1, \ldots, m-1\}}\left\{u_{k}-u_{k+1}\right\}}{3}$, the probability that there exists $\hat{n} \in \mathbb{N}$ such that $\bar{u}_{k}\left(v^{n}\right) \in$ $\left(u_{k}-d, u_{k}+d\right)$ for any $n>\hat{n}$ and for each alternative $k$ is one.

Let $V \subset[0,1]^{\infty \times m}$ denote the subset of realizations of matrix $v$ such that $\lim _{n \rightarrow \infty} \bar{u}_{k}\left(v^{n}\right)=$ $u_{k}$. By Expression (3), $\operatorname{Pr}[v \in V]=1$.

Hereafter, we consider realizations of $v$ such that $v \in V$.

For any $v \in V$, we consider the sequence of games $\left\{\Gamma\left(v^{n}\right)\right\}_{n=2}^{\infty}$. Let $a^{*}(v) \equiv\left\{a^{*}\left(v^{n}\right)\right\}_{n=2}^{\infty} \in$ $\prod_{n=2}^{\infty}[-1,1]^{n \times m}$ denote a sequence of Nash equilibria corresponding to the sequence of games $\left\{\Gamma\left(v^{n}\right)\right\}_{n=2}^{\infty}$. Then, for each $n \in \mathbb{N} \backslash\{1\}$, and for each $i \in N(n), a^{*, i}\left(v^{n}\right) \in[-1,1]^{m}$ de- 
notes agent $i^{\prime} s$ action vector in the equilibrium $a^{*}\left(v^{n}\right), A_{k}^{*}\left(v^{n}\right) \equiv \sum_{i=1}^{n} a_{k}^{*, i}\left(v^{n}\right)$ and $A^{*}\left(v^{n}\right) \equiv$ $\left(A_{1}^{*}\left(v^{n}\right), \ldots, A_{m}^{*}\left(v^{n}\right)\right) \in[-n, n]^{m}$.

In the Appendix, we prove several intermediate results en route to our main proposition. Namely, for any $v \in V$, for any sequence of equilibria $a^{*}(v) \in \prod_{n=2}^{\infty}[-1,1]^{m \times n}$, if the size $n$ of the subset of participating agents $\{1, \ldots, n\}$ is sufficiently large:

i) one alternative wins with probability arbitrarily close to one (Lemma 3 and Corollary $2)$. We call this alternative the "likely winner" and we denote it $w\left(a^{*}(v)\right)$;

ii) alternatives that are better than the likely winner receive strictly positive net aggregate actions, and alternatives that are worse than the likely winner receive strictly negative net aggregate actions (Lemma 4);

iii) if the likely winner is not the best alternative, then the net aggregate actions for the likely winner diverge toward infinity (Lemma 5), and at the same time, the net aggregate actions for some other alternative worse than the likely winner decrease unboundedly toward minus infinity, so the likely winner cannot be the worst alternative (Lemma 6 and Corollary $3)$;

iv) and in fact, the net aggregate actions for all other alternatives worse than the likely winner decrease unboundedly toward minus infinity (Lemma 7 and Corollary 4).

We seek to prove that the probability that the chosen alternative is the one that maximizes social welfare converges to one in the number of agents who participate in the quadratic voting mechanism. To formally state this result, we require additional definitions. Given an equilibrium $a^{*}\left(v^{n}\right) \in[-1,1]^{n \times m}$ for each game $\Gamma\left(v^{n}\right)$ for each $n \in \mathbb{N} \backslash\{1\}$, and given the corresponding sequence of equilibria $\left\{a^{*}\left(v^{n}\right)\right\}_{n=1}^{\infty} \in \prod_{n=2}^{\infty}[-1,1]^{n \times m}$ for each realization of the matrix of values $v \in[0,1]^{\mathbb{N} \times m}$, let

$$
a^{*} \in\left(\prod_{n=2}^{\infty}[-1,1]^{n \times m}\right)^{[0,1]^{\mathbb{N} \times m}}
$$

be the collection of equilibria, unconditional on $v$, so $a^{*}$ specifies an equilibrium to be played for any possible realization of the matrix of values $v$ and any value of the size $n$. Let $\mathcal{A}^{*}$ denote the family of all such collections of equilibria.

For any $n \in \mathbb{N} \backslash\{1\}$ and any $v^{n} \in[0,1]^{n \times m}$, let $b\left(v^{n}\right) \subseteq X$ denote the set of best alterna- 
tives, defined by

$$
b\left(v^{n}\right) \equiv \arg \max _{k \in X} \bar{u}_{k}\left(v^{n}\right) .
$$

These are the alternatives that maximize aggregate values. Given any collection of unconditional equilibria $a^{*} \in \mathcal{A}^{*}$, for each game $\Gamma\left(v^{n}\right)$, let $x\left(v^{n}, a^{*}\right) \in X$ denote the chosen alternative in $\Gamma\left(v^{n}\right)$.

Uncertainty over the realization of $v$ introduces uncertainty about the outcome $x\left(v^{n}, a^{*}\right)$. Recall that for each agent $i \in \mathbb{N}$, the vector $u^{i}=\left(u_{1}^{i}, \ldots, u_{m}^{i}\right)$, in which the $k$-th component is the value that agent $i$ assigns to alternative $k$, is drawn independently for each $i \in \mathbb{N}$ from a distribution $F$ over $[0,1]^{m}$. These independent draws from $F$ induce a probability distribution over the infinite matrix of values $v$, and hence, on the probability that each particular sequence of games $\left\{\Gamma\left(v^{n}\right)\right\}_{n=2}^{\infty}$ is played, and, given $a^{*}$, that the sequence $\left\{x\left(v^{n}, a^{*}\right)\right\}_{n=2}^{\infty}$ are chosen. Namely, if we let $\bar{v}$ denote the infinite matrix of values as a random variable, and $v \in[0,1]^{\mathbb{N} \times m}$ as its realization, the probability that the sequence $\left\{x\left(v^{n}, a^{*}\right)\right\}_{n=2}^{\infty}$ of alternatives are chosen is $\operatorname{Pr}[\bar{v}=v]$.

An ideal collective choice mechanism would be one such that for any realization of $v$, and for any equilibrium of the game induced by the mechanism given $v$, the outcome is a best alternative. That is, an ideal mechanism would deliver $x\left(v^{n}, a^{*}\right) \in b\left(v^{n}\right)$ for any $n \in \mathbb{N} \backslash\{1\}$ and for any $v \in[0,1]^{\mathbb{N} \times m}$. Since no budget-balanced mechanism achieves this goal (see for instance, Proposition 5.1 and Corollary 5.1 in Börgers 2015), consider a second best: a mechanism that makes a best choice with probability converging to one as the population participating in the collective choice becomes arbitrarily large. While such a mechanism is not be infallible, its misses become vanishingly rare. We say that such a mechanism is asymptotically optimal.

Definition 1 A collective choice mechanism is asymptotically optimal if

$$
\lim _{n \longrightarrow \infty} \operatorname{Pr}\left[x\left(v^{n}, a^{*}\right) \in b\left(v^{n}\right)\right]=1 \text { for any } a^{*} \in \mathcal{A}^{*} .
$$

An asymptotically optimal mechanism chooses the best alternative with probability converging to one, for any collection $a^{*}$ of sequences of equilibria. The uncertainty is with respect to the realization of the matrix of values $v$, and with respect to any stochastic element in the choice inherent in the rules of the mechanism.

Since values are bounded, an asymptotically optimal mechanism also asymptotically maximizes social welfare, calculated as the average value of the chosen alternative, across all 
agents.

Proposition 1 The quadratic voting mechanism is asymptotically optimal.

The definition of asymptotic optimality is ex-ante, in the sense that the optimality of the mechanism is assessed before the matrix of values $v$ is known. We can also define asymptotic optimality ex-post, for each given realization of the infinite value matrix $v \in[0,1]^{\mathbb{N} \times m}$.

Definition 2 For any $v \in[0,1]^{\mathbb{N} \times m}$, a collective choice mechanism is ex-post asymptotically optimal for $v$ if $\lim _{n \rightarrow \infty} \operatorname{Pr}\left[x\left(v^{n}, a^{*}\right) \in b\left(v^{n}\right)\right]=1$ for any sequence of equilibria $\left\{a^{*}(v)\right\}_{n=2}^{\infty}$.

Let $\hat{V} \subseteq[-1,1]^{\mathbb{N} \times m}$ denote the subset of infinite value matrixes for which the mechanism is ex-post asymptotically optimal. We say the mechanism is generically ex-post asymptotically optimal if $\operatorname{Pr}[v \in \hat{V}]=1$, and that it is ex-post asymptotically optimal if it is ex-post asymptotically optimal for $v$, for any $v \in[-1,1]^{\mathbb{N} \times m}$.

A mechanism that is ex-post asymptotically optimal for $v$ chooses the best alternative with probability converging to one, given $v$, for any equilibria. Since values are known, the uncertainty is only with respect to any stochastic element in the choice inherent in the rules of the mechanism. The mechanism is generically ex-post asymptotically optimal if it is expost asymptotically optimal for a generic value matrix $v$, where genericity of a set is defined as probability measure one.

Corollary 1 The quadratic voting mechanism is generically ex-post asymptotically optimal: it is ex-post asymptotically optimal for $v$, for any $v \in V^{9}$

We show by example that convergence to optimality can be fast.

Example 1 Assume the set of alternatives is $\{1,2,3\}$, and $u^{i}=\left\{\begin{array}{l}\left(1, \frac{2}{3}, 0\right) \text { for any odd } i \in \mathbb{N} \\ \left(0, \frac{2}{3}, 1\right) \text { for any even } i \in \mathbb{N}\end{array}\right.$. The unique equilibrium is such that the probability that the ex-post utilitarian optimum (alternative 2) is chosen with 95\% probability if $n=100$, and with 99\% probability if $n=1,000$.

\footnotetext{
${ }^{9}$ We established that quadratic voting is ex-post asymptotically optimal for any $v \in V$ as part of the proof that it is ex-ante asymptotically optimal. And $v \in V$ holds with probability one by the law of large numbers, as we observed in Expression (3).
} 
Besides optimality, the mechanism also satisfies the property of asymptotically requiring zero transfers across agents: as the number of participants increases, the equilibrium action of each agent converges to zero, so her contribution converges to zero as well, and thus the per capita amount collected and redistributed by the mechanism converges to zero.

Proposition 2 For any $v \in V$, and for any sequence of equilibria $a^{*}(v)$, and for any $i \in \mathbb{N}$, $\lim _{n \longrightarrow \infty} a^{*, i}\left(v^{n}\right) \longrightarrow 0$, and thus agent $i^{\prime} s$ monetary contribution to the mechanism converges to zero.

Proposition 2 guarantees that even if some agents are budget constrained, however low their constraint, as long as this constraint is strictly positive, a sufficiently large society can run the quadratic voting mechanism with full participation by all agents.

Our last result strengthens Proposition 1, to also show that not only the best alternative receives most votes, but in addition, the full vote tally is ordered from best to worst alternatives, so that the second best alternative receives the second most net votes, the third best comes third, and so on.

Proposition 3 There exists $\hat{t} \in \mathbb{N}$ such that for any alternative $k \in\{1, \ldots, m-1\}$, and for any $t>\hat{t}, A_{k}^{*}\left(v^{n(t)}\right)>A_{k+1}^{*}\left(v^{n(t)}\right)$.

It follows that if society is sufficiently large, even in the vanishingly rare event in which the quadratic voting mechanism does not choose optimally, for any two alternatives, the mechanism is strictly more likely to choose the socially better one.

We have proposed a quadratic voting mechanism for a collective choice with multiple alternatives. The mechanism allows each agent to express an intensity of preference for or against each alternative, and charges the agent for each alternative, the square of the agent's expressed intensity.

We highlight two assumptions that limit the scope of appropriate applications: risk neutrality, and agents' common knowledge about valuations.

The assumption that agents' are risk neutral, albeit standard in mechanism design, is undoubtedly restrictive. As we note in the introduction, risk-neutrality is an appropriate assumption in applications in which the agents are firms or investors who maximize expected 
profits. Note that because transfers vanish to zero, the assumption of risk-neutrality is also innocuous in applications in which agents are risk-averse but they all have the same wealth. Whereas, in applications in which agents are risk-averse and the wealth distribution is unequal, the interpretation of the result is more fraught: if the marginal value of wealth is lower for wealthier agents, then wealthier agents will value the choice (in wealth units) more than poor ones, so the collective choice will be disproportionally responsive toward wealthier agents.

With regard to information, we assume that agents' valuations are common-knowledge among agents. Under this information assumption, our result that the vote-buying mechanism with quadratic costs is asymptotically optimal amounts to the finding that the mechanism asymptotically implements in Nash equilibrium the social choice function that maximizes utilitarian welfare. This result is robust if we weaken the assumption of common knowledge of values, to assume that only the distribution of value vectors, and not the precise value matrix, is common knowledge.

With these assumptions, our main result is that the mechanism is asymptotically optimal (Proposition 1). In addition we have established that transfers converge to zero (Proposition 2) and that the probability that each alternative is chosen is asymptotically ranked in accordance to its social efficiency (Proposition 3). An appendix follows, where we prove our results.

\section{Appendix}

Lemma 1 There exists a $\bar{c} \in \mathbb{R}_{++}$such that, if $c>\bar{c}$, for any $v \in[0,1]^{\mathbb{N} \times m}$, and for any $n \in \mathbb{N} \backslash\{1\}$, a pure Nash equilibrium of game $\Gamma\left(v^{n}\right)$ exists.

Proof. For any $v \in[0,1]^{\mathbb{N} \times m}$, and for any $n \in \mathbb{N} \backslash\{1\}$, game $\Gamma\left(v^{n}\right)$ has finitely many players (namely, it has $n$ players). Strategy spaces for each player $i$ in game $\Gamma\left(v^{n}\right)$ are $[-1,1]^{m}$, hence non-empty, convex, compact subsets of an Euclidean space. Further, the payoff function $U^{i}$ is continuous in $a\left(v^{n}\right)$ for each $i \in\{1, . ., n\}$. If $U^{i}$ is concave (and hence quasiconcave) in $a^{i}\left(v^{n}\right)$ for each $i \in\{1, \ldots, n\}$, then a pure strategy equilibrium exists (Debreu 1952).

Therefore, to prove there exists a $\bar{c} \in \mathbb{R}_{++}$such that, if $c>\bar{c}$, for any $v \in[0,1]^{\mathbb{N} \times m}$, and for any $n \in \mathbb{N} \backslash\{1\}$, a pure Nash equilibrium of game $\Gamma\left(v^{n}\right)$ exists, it suffices to show that there exists a $\bar{c} \in \mathbb{R}_{++}$such that, if $c>\bar{c}$, for any $v \in[0,1]^{\mathbb{N} \times m}$, and for any $n \in \mathbb{N} \backslash\{1\}$, the 
utility function $U^{i}$ is concave in $a^{i}\left(v^{n}\right)$ for each $i \in\{1, \ldots, n\}$. This is what we show.

Note first that the concavity of $U^{i}$ is the same as the concavity of $\hat{U}^{i} \equiv \frac{1}{c} U^{i}$. So it suffices to establish that $\hat{U}^{i}$ is concave in $a^{i}\left(v^{n}\right)$ for each $i \in\{1, \ldots, n\}$. We construct the Hessian $H\left(\hat{U}^{i}\left(a\left(v^{n}\right)\right)\right)$. Note

$$
\begin{aligned}
\frac{\partial \hat{U}^{i}\left(a\left(v^{n}\right)\right)}{\partial a_{k}^{i}\left(v^{n}\right)} & =\sum_{h=1}^{m} \frac{\partial}{\partial a_{k}^{i}\left(v^{n}\right)} p_{h}\left(A\left(v^{n}\right)\right) \frac{u_{h}^{i}}{c}-2 a_{k}^{i}\left(v^{n}\right), \\
\frac{\partial^{2} \hat{U}^{i}\left(a\left(v^{n}\right)\right)}{\partial a_{k}^{i}\left(v^{n}\right) \partial a_{k}^{i}\left(v^{n}\right)} & =\sum_{h=1}^{m} \frac{\partial^{2}}{\left(\partial a_{k}^{i}\left(v^{n}\right)\right)^{2}} p_{h}\left(A\left(v^{n}\right)\right) \frac{u_{h}^{i}}{c}-2, \\
\frac{\partial^{2} \hat{U}^{i}\left(a\left(v^{n}\right)\right)}{\partial a_{k}^{i}\left(v^{n}\right) \partial a_{l}^{i}\left(v^{n}\right)} & =\sum_{h=1}^{m} \frac{\partial^{2}}{\partial a_{k}^{i}\left(v^{n}\right) \partial a_{l}^{i}\left(v^{n}\right)} p_{h}\left(A\left(v^{n}\right)\right) \frac{u_{h}^{i}}{c}
\end{aligned}
$$

and

$$
\begin{aligned}
\frac{\partial}{\partial a_{k}^{i}\left(v^{n}\right)} p_{k}\left(A\left(v^{n}\right)\right) & =\frac{e^{A_{k}\left(v^{n}\right)} \sum_{h=1}^{m} e^{A_{h}\left(v^{n}\right)}-\left(e^{A_{k}\left(v^{n}\right)}\right)^{2}}{\left(\sum_{h=1}^{m} e^{A_{h}\left(v^{n}\right)}\right)^{2}}=p_{k}\left(A\left(v^{n}\right)\right)-\left(p_{k}\left(A\left(v^{n}\right)\right)\right)^{2} \\
\frac{\partial}{\partial a_{k}^{i}\left(v^{n}\right)} p_{l}\left(A\left(v^{n}\right)\right) & =\frac{\partial}{\partial a_{l}^{i}\left(v^{n}\right)} p_{k}\left(A\left(v^{n}\right)\right)=\frac{-e^{A_{l}\left(v^{n}\right)} e^{A_{k}\left(v^{n}\right)}}{\left(\sum_{h=1}^{m} e^{A_{h}\left(v^{n}\right)}\right)^{2}}=-p_{l}\left(A\left(v^{n}\right)\right) p_{k}\left(A\left(v^{n}\right)\right), \\
\frac{\partial^{2}}{\partial a_{k}^{i}\left(v^{n}\right) \partial a_{k}^{i}\left(v^{n}\right)} p_{k}\left(A\left(v^{n}\right)\right) & =p_{k}\left(A\left(v^{n}\right)\right)-\left(p_{k}\left(A\left(v^{n}\right)\right)\right)^{2}-2 p_{k}\left(A\left(v^{n}\right)\right)\left(p_{k}\left(A\left(v^{n}\right)\right)-\left(p_{k}\left(A\left(v^{n}\right)\right)\right)^{2}\right) \\
\frac{\partial^{2}}{\partial a_{k}^{i}\left(v^{n}\right) \partial a_{l}^{i}\left(v^{n}\right)} p_{k}\left(A\left(v^{n}\right)\right) & =-p_{l}\left(A\left(v^{n}\right)\right) p_{k}\left(A\left(v^{n}\right)\right)+2 p_{l}\left(A\left(v^{n}\right)\right)\left(p_{k}\left(A\left(v^{n}\right)\right)\right)^{2}, \\
\frac{\partial^{2}}{\partial a_{l}^{i}\left(v^{n}\right) \partial a_{h}^{i}\left(v^{n}\right)} p_{k}\left(A\left(v^{n}\right)\right) & =-2 p_{l}\left(A\left(v^{n}\right)\right) p_{h}\left(A\left(v^{n}\right)\right) p_{k}\left(A\left(v^{n}\right)\right), \\
\frac{\partial^{2}}{\partial a_{l}^{i}\left(v^{n}\right) \partial a_{l}^{i}\left(v^{n}\right)} p_{k}\left(A\left(v^{n}\right)\right) & =-\left(p_{l}\left(A\left(v^{n}\right)\right)-\left(p_{l}\left(A\left(v^{n}\right)\right)\right)^{2}\right) p_{k}\left(A\left(v^{n}\right)\right)-p_{l}\left(A\left(v^{n}\right)\right)\left(-p_{l}\left(A\left(v^{n}\right)\right) p_{k}\left(A\left(v^{n}\right)\right)\right) \\
& =p_{l}\left(A\left(v^{n}\right)\right) p_{k}\left(A\left(v^{n}\right)\right)\left(2 p_{l}\left(A\left(v^{n}\right)\right)-1\right)
\end{aligned}
$$

Since $p_{k}\left(A\left(v^{n}\right)\right) \in(0,1)$ for any $k \in\{1, \ldots, m\}$ and for any $A\left(v^{n}\right)$, it follows that $\frac{\partial^{2}}{\partial a_{k}^{i}\left(v^{n}\right) \partial a_{k}^{i}\left(v^{n}\right)} p_{k}\left(A\left(v^{n}\right)\right)$, $\frac{\partial^{2}}{\partial a_{k}^{i}\left(v^{n}\right) \partial a_{l}^{i}\left(v^{n}\right)} p_{k}\left(A\left(v^{n}\right)\right), \frac{\partial^{2}}{\partial a_{l}^{i}\left(v^{n}\right) \partial a_{h}^{i}\left(v^{n}\right)} p_{k}\left(A\left(v^{n}\right)\right)$ and $\frac{\partial^{2}}{\partial a_{l}^{i}\left(v^{n}\right) \partial a_{l}^{i}\left(v^{n}\right)} p_{k}\left(A\left(v^{n}\right)\right)$ are bounded below by -2 and above by 1 .

Therefore, $\sum_{h=1}^{m} \frac{\partial^{2}}{\left(\partial a_{k}^{i}\left(v^{n}\right)\right)^{2}} p_{h}\left(A\left(v^{n}\right)\right) u_{h}^{i}$ and $\sum_{h=1}^{m} \frac{\partial^{2}}{\partial a_{k}^{i}\left(v^{n}\right) \partial a_{l}^{i}\left(v^{n}\right)} p_{h}\left(A\left(v^{n}\right)\right) u_{h}^{i}$ are bounded 
below by $-2 m$ and above by $2 m$. Hence

$$
\begin{aligned}
\lim _{c \longrightarrow \infty} \sup _{n \in \mathbb{N} \backslash\{1\}}\left|\frac{\partial^{2} \hat{U}^{i}\left(a\left(v^{n}\right)\right)}{\partial a_{k}^{i}\left(v^{n}\right) \partial a_{k}^{i}\left(v^{n}\right)}-2\right| & =0, \text { and } \\
\lim _{c \longrightarrow \infty} \sup _{n \in \mathbb{N} \backslash\{1\}}\left|\frac{\partial^{2} \hat{U}^{i}\left(a\left(v^{n}\right)\right)}{\partial a_{k}^{i}\left(v^{n}\right) \partial a_{l}^{i}\left(v^{n}\right)}\right| & =0 .
\end{aligned}
$$

Then $\lim _{c \longrightarrow \infty} \sup _{n \in \mathbb{N} \backslash\{1\}}\left|M_{k}(n)-(2)^{k}\right|=0$, where $M_{k}\left(v^{n}\right)$ denotes the $k$ - th leading principal minor of the Hessian $H\left(\hat{U}^{i}\left(a\left(v^{n}\right)\right)\right)$. Hence, there exists $\bar{c} \in \mathbb{R}_{++}$such that for any $c>\bar{c}$, and for any $n \in \mathbb{N} \backslash\{1\}, H\left(\hat{U}^{i}\left(a\left(v^{n}\right)\right)\right.$ is negative definite, and thus $\hat{U}^{i}\left(a\left(v^{n}\right)\right)$ is strictly concave (see, for instance, Simon and Blume 1994, Thm 21.5).

Since the concavity of a function is preserved under scaling, it follows that for any $c>\bar{c}$, and for any $n \in \mathbb{N} \backslash\{1\}, H\left(U^{i}\left(a\left(v^{n}\right)\right)\right.$ is also negative definite, and thus $U^{i}\left(a\left(v^{n}\right)\right)$ is also strictly concave.

Thus, for any $c>\bar{c}$, a pure strategy equilibrium exists (Debreu 1952) for any game $\Gamma\left(v^{n}\right)$.

Lemma 2 For each $v \in[0,1]^{\mathbb{N} \times m}$, for each $n \in \mathbb{N} \backslash\{1\}$, and for each equilibrium $a^{*}\left(v^{n}\right) \in$ $[-1,1]^{n \times m}$ of game $\Gamma\left(v^{n}\right), a_{k}^{*, i}\left(v^{n}\right) \in(-1,1)$ for each $i \in\{1, \ldots, n\}$ and each $k \in X$.

Proof. In each game $\Gamma\left(v^{n}\right)$, for each $i \in\{1, \ldots, n\}$ and for each alternative $k \in X$, from the utility function (2), the marginal utility for agent $i$ of action $k$ is (from expressions (4), (5) and (6) in the proof of Lemma 1)

$$
\begin{aligned}
\frac{\partial}{\partial a_{k}^{i}} U^{i}\left(a^{*}\left(v^{n}\right)\right) & =\sum_{h=1}^{m} \frac{\partial}{\partial a_{k}^{i}\left(v^{n}\right)} p_{h}\left(A\left(v^{n}\right)\right) u_{h}^{i}-2 c a_{k}^{i}\left(v^{n}\right) \\
& =p_{k}\left(A^{*}\left(v^{n}\right)\right) u_{k}^{i}\left(1-p_{k}\left(A^{*}\left(v^{n}\right)\right)\right)-\sum_{h \in X \backslash\{k\}} p_{k}\left(A^{*}\left(v^{n}\right)\right) p_{h}\left(A^{*}\left(v^{n}\right)\right) u_{h}^{i}-2 c a_{k}^{i}\left(v^{n}\right) \\
& =p_{k}\left(A^{*}\left(v^{n}\right)\right) \sum_{h \in X \backslash\{k\}}\left(u_{k}^{i}-u_{h}^{i}\right) p_{h}\left(A^{*}\left(v^{n}\right)\right)-2 c a_{k}^{i}\left(v^{n}\right) .
\end{aligned}
$$

Since $u_{k}^{i}-u_{h}^{i} \in[-1,1]$, the absolute value of first term is bounded above by one. Since by assumption $c>\frac{1}{2}$, at the corner $a_{k}^{i}\left(v^{n}\right)=-1,-2 c a_{k}^{i}\left(v^{n}\right)>1$ so the marginal utility of action $a_{k}^{i}\left(v^{n}\right)$ is strictly positive, so $a_{k}^{i}\left(v^{n}\right)=-1$ is not part of an equilibrium; similarly, at the corner $a_{k}^{i}\left(v^{n}\right)=1,-2 c a_{k}^{i}\left(v^{n}\right)<-1$ and the marginal utility is negative so the positive 
corner is not part of an equilibrium. Hence, any equilibrium is interior.

We establish a first asymptotic result: in any equilibrium in a game with sufficiently many players, there is one alternative that wins with arbitrarily large probability.

Lemma 3 For any $v \in V$, for any sequence of equilibria $a^{*}(v) \in \prod_{n=2}^{\infty}[-1,1]^{m \times n}$, and for any $\varepsilon \in \mathbb{R}_{++}$, there exists $n(\varepsilon) \in \mathbb{N}$ such that, for any $n>n(\varepsilon)$, there is $k\left(n, a^{*}(v)\right) \in\{1, \ldots, m\}$ such that $p_{k\left(n, a^{*}(v)\right)}\left(A^{*}\left(v^{n}\right)\right)>1-\varepsilon$.

Proof. Since $v \in V, \lim _{n \longrightarrow \infty} \bar{u}_{k}\left(v^{n}\right)=u_{k}$.

Given that the number of alternatives is finite, for any $n \in \mathbb{N}$ such that $n>\hat{n}$, and for any equilibrium $a^{*}\left(v^{n}\right)$ of game $\Gamma\left(v^{n}\right)$, there is at least one $k\left(n, a^{*}(v)\right) \in\{1, \ldots, m\}$ such that $\left.p_{k\left(n, a^{*}(v)\right)}\left(A^{*}\left(v^{n}\right)\right)\right)>\varepsilon$, for every $\varepsilon \in\left(0, \frac{1}{m}\right)$.

We prove the lemma by contradiction. Given a sequence of equilibria $a^{*}(v) \in \prod_{n=\hat{n}}^{\infty}[-1,1]^{m \times n}$, assume (absurd) that there exists $\varepsilon \in \mathbb{R}_{++}$and a strictly increasing $\tilde{n}: \mathbb{N} \longrightarrow \mathbb{N}$ such that, for each $\tau \in \mathbb{N}$, there is a set of alternatives $K(\tau) \subset\{1,2, \ldots, m\}$ with $|K(\tau)|>1$, such that $p_{k}\left(A^{*}\left(v^{\tilde{n}(\tau)}\right)>\varepsilon \Longleftrightarrow k \in K(\tau)\right.$.

Since the set of alternatives is finite, this assumption implies that there exists $\varepsilon \in \mathbb{R}_{++}$ and a strictly increasing $n^{\prime}: \mathbb{N} \longrightarrow \mathbb{N}$ such that, for each $\tau \in \mathbb{N}$, there is a set of alternatives $K \subset\{1,2, \ldots, m\}$ with $|K|>1$, such that $p_{k}\left(A^{*}\left(v^{n^{\prime}(\tau)}\right)>\varepsilon \Longleftrightarrow k \in K{ }^{10}\right.$

Let $K^{+} \subseteq X$ be the largest set of alternatives such that there exists $\delta \in(0,1)$ and a strictly increasing $n^{\prime}: \mathbb{N} \longrightarrow \mathbb{N}$ such that, for each $\tau \in \mathbb{N}$,

$$
p_{k}\left(A^{*}\left(v^{n^{\prime}(\tau)}\right)>\delta \Longleftrightarrow k \in K^{+}\right.
$$

Since $|K|>1$ and $K$ satisfies the conditions in this definition, $\left|K^{+}\right| \geq|K|$ so $\left|K^{+}\right|>1$.

Note that for any $h \in X \backslash K^{+}, \lim _{\tau \longrightarrow \infty} p_{h}\left(A^{*}\left(v^{n^{\prime}(\tau)}\right)\right)=0$; otherwise (if the limit does not exist or is not zero), then there would exist an $h \in X \backslash K^{+}$and a subsequence $\tau: \mathbb{N} \longrightarrow \mathbb{N}$ and a $\delta^{\prime} \in(0, \delta)$ such that $p_{h}\left(A^{*}\left(v^{n^{\prime}(\tau(\gamma))}\right)\right)>\delta^{\prime}$ for any $\gamma \in \mathbb{N}$, and then there would also

\footnotetext{
${ }^{10}$ Note $\left\{n^{\prime}(\tau)\right\}_{\tau=1}^{\infty}$ is a subsequence of $\{\tilde{n}(\tau)\}_{\tau=1}^{\infty}$, but we do not denote it as such, to save on notation.
} 
exist a $K^{++} \supseteq K^{+} \cup\{h\}$ such that for each $\gamma \in \mathbb{N}, p_{k}\left(A^{*}\left(v^{n^{\prime}(\tau(\gamma))}\right)\right)>\delta^{\prime} \Longleftrightarrow k \in K^{++}$, but this is impossible by definition of $K^{+}$. Hence, for any $h \in X \backslash K^{+}, \lim _{\tau \longrightarrow \infty} p_{h}\left(A^{*}\left(v^{n^{\prime}(\tau)}\right)\right)=0$.

For any $n \in \mathbb{N} \backslash\{1\}$, define $\bar{u}\left(v, a^{*}\left(v^{n}\right)\right) \equiv \sum_{h=1}^{m} p_{h}\left(A^{*}\left(v^{n}\right) \bar{u}_{h}\left(v^{n}\right)\right.$. This is expected value of the chosen alternative, averaged across all agents in $\{1, \ldots, n\}$, according to equilibrium $a^{*}$, and given the realization of the matrix of values $v^{n}$. Let $k_{\max } \in \arg \max _{k \in K^{+}} u_{k}, k_{\min } \in \arg \min _{k \in K^{+}} u_{k}$. Then, since $k_{\max } \in K^{+}$and $k_{\min } \in K^{+}$, by definition of $K^{+}, p_{k_{\max }}\left(a^{*}\left(v^{n^{\prime}(\tau)}\right)\right)>\delta \in(0,1)$ and $p_{k_{\min }}\left(a^{*}\left(v^{n^{\prime}(\tau)}\right)\right)>\delta \in(0,1)$ for any $\tau \in \mathbb{N}$, and since $\lim _{\tau \longrightarrow \infty} p_{h}\left(A^{*}\left(v^{n^{\prime}(\tau)}\right)\right)=0$ for any $h \in X \backslash K^{+}$, it follows that there exists $\hat{\tau} \in \mathbb{N}$ such that

$$
\bar{u}_{k_{\max }}\left(v^{n^{\prime}(\tau)}\right)>\bar{u}\left(v^{n^{\prime}(\tau)}\right)>\bar{u}_{k_{\min }}\left(v^{n^{\prime}(\tau)}\right)
$$

for any $\tau>\hat{\tau}$.

Further, since

$$
p_{k_{\max }}\left(a^{*}\left(v^{n^{\prime}(\tau)}\right)\right)>\delta \text { and } p_{k_{\min }}\left(a^{*}\left(v^{n^{\prime}(\tau)}\right)\right)>\delta
$$

it follows that

$$
\frac{p_{k_{\max }}\left(a^{*}\left(v^{n^{\prime}(\tau)}\right)\right)}{p_{k_{\min }}\left(a^{*}\left(v^{n^{\prime}(\tau)}\right)\right)} \in\left(\frac{\delta}{1-\delta}, \frac{1-\delta}{\delta}\right) .
$$

Since any equilibrium is interior (Lemma 2), for any $v \in V$ and any $n \in \mathbb{N} \backslash\{1\}$, any equilibrium $a^{*}\left(v^{n}\right)$ of game $\Gamma\left(v^{n}\right)$ satisfies the First Order Condition:

$$
\frac{\partial}{\partial a_{k}^{i}} U^{i}\left(a^{*}\left(v^{n}\right)\right)=0
$$

for each $k \in X$ and for each $i \in\{1, \ldots, n\}$. We can restate the first order condition as follows:

$$
\begin{aligned}
\sum_{h=1}^{m} \frac{\partial}{\partial a_{k}^{i}} p_{h}\left(A^{*}\left(v^{n}\right)\right) u_{h}^{i}-2 c a_{k}^{*, i}\left(v^{n}\right) & =0 \\
p_{k}\left(A^{*}\left(v^{n}\right)\right) u_{k}^{i}\left(1-p_{k}\left(A^{*}\left(v^{n}\right)\right)\right)-\sum_{h \in X \backslash\{k\}} p_{k}\left(A^{*}\left(v^{n}\right)\right) p_{h}\left(A^{*}\left(v^{n}\right)\right) u_{h}^{i} & =2 c a_{k}^{*, i}\left(v^{n}\right) \\
\frac{p_{k}\left(A^{*}\left(v^{n}\right)\right)}{2 c} \sum_{h \in X \backslash\{k\}}\left(u_{k}^{i}-u_{h}^{i}\right) p_{h}\left(A^{*}\left(v^{n}\right)\right) & =a_{k}^{*, i}\left(v^{n}\right) .
\end{aligned}
$$

Note that from the First Order Condition as restated in Expression (10), and summing across 
all agents,

$$
\begin{aligned}
A_{k}^{*}\left(v^{n}\right) & =\sum_{i=1}^{n} \frac{p_{k}\left(A^{*}\left(v^{n}\right)\right)}{2 c} \sum_{h \in X \backslash\{k\}}\left(u_{k}^{i}-u_{h}^{i}\right) p_{h}\left(A^{*}\left(v^{n}\right)\right) \\
& =\sum_{i=1}^{n} \frac{p_{k}\left(A^{*}\left(v^{n}\right)\right)}{2 c} \sum_{h \in X \backslash\{k\}} u_{k}^{i} p_{h}\left(A^{*}\left(v^{n}\right)\right)-\sum_{i=1}^{n} \frac{p_{k}\left(A^{*}\left(v^{n}\right)\right)}{2 c} \sum_{h \in X \backslash\{k\}} u_{h}^{i} p_{h}\left(A^{*}\left(v^{n}\right)\right) \\
& =\frac{p_{k}\left(A^{*}\left(v^{n}\right)\right)}{2 c} \sum_{i=1}^{n} u_{k}^{i} \sum_{h \in X \backslash\{k\}} p_{h}\left(A^{*}\left(v^{n}\right)\right)-\frac{p_{k}\left(A^{*}\left(v^{n}\right)\right)}{2 c} \sum_{i=1}^{n} \sum_{h \in X \backslash\{k\}} u_{h}^{i} p_{h}\left(A^{*}\left(v^{n}\right)\right) \\
& =\frac{p_{k}\left(A^{*}\left(v^{n}\right)\right) n}{2 c} \bar{u}_{k}\left(v^{n}\right) \sum_{h \in X \backslash\{k\}} p_{h}\left(A^{*}\left(v^{n}\right)\right)-\frac{p_{k}\left(A^{*}\left(v^{n}\right)\right)}{2 c} n \sum_{h \in X \backslash\{k\}} \bar{u}_{h}\left(v^{n}\right) p_{h}\left(A^{*}\left(v^{n}\right)\right) \\
& =\frac{p_{k}\left(A^{*}\left(v^{n}\right)\right) n}{2 c} \sum_{h \in X}\left(\bar{u}_{k}\left(v^{n}\right)-\bar{u}_{h}\left(v^{n}\right)\right) p_{h}\left(A^{*}\left(v^{n}\right)\right),
\end{aligned}
$$

which implies

$$
\begin{aligned}
& \frac{p_{k_{\max }}\left(a^{*}\left(v^{n^{\prime}(\tau)}\right)\right)}{p_{k_{\min }}\left(a^{*}\left(v^{n^{\prime}(\tau)}\right)\right)}=\frac{\frac{\exp \left(A_{k_{\max }^{*}}^{*}\left(v^{\left.n^{\prime}(\tau)\right)}\right)\right.}{\left.\sum_{h \in X} A_{h}^{*}\left(v^{n^{\prime}(\tau)}\right)\right)}}{\frac{\exp \left(A_{k_{\min }^{*}}^{*}\left(v^{n^{\prime}(\tau)}\right)\right)}{\left(\sum_{h \in X} A_{h}^{*}\left(v^{n^{\prime}(\tau)}\right)\right)}}=\frac{\exp \left(\frac{p_{k_{\max }}\left(A^{*}\left(v^{n^{\prime}(\tau)}\right)\right) \cdot n^{\prime}(\tau)}{2 c} \sum_{h \in X}\left(\bar{u}_{k_{\max }}\left(v^{n^{\prime}(\tau)}\right)-\bar{u}_{h}\left(v^{n^{\prime}(\tau)}\right)\right) p_{h}\left(A^{*}\left(v^{n^{\prime}(\tau)}\right)\right)\right)}{\exp \left(\frac{p_{k_{\min }}\left(A^{*}\left(v^{n^{\prime}(\tau)}\right)\right) \cdot n^{\prime}(\tau)}{2 c} \sum_{h \in X}\left(\bar{u}_{k_{\min }}\left(v^{n^{\prime}(\tau)}\right)-\bar{u}_{h}\left(v^{n^{\prime}(\tau)}\right)\right) p_{h}\left(A^{*}\left(v^{n^{\prime}(\tau)}\right)\right)\right)} \\
& =\frac{\exp \left(\frac{p_{k_{\max }}\left(A^{*}\left(v^{n^{\prime}}(\tau)\right)\right) \cdot n^{\prime}(\tau)}{2 c}\left(\bar{u}_{k_{\max }}\left(v^{n^{\prime}(\tau)}\right)-\bar{u}\left(v^{n^{\prime}(\tau)}\right)\right)\right)}{\exp \left(\frac{p_{k_{\min }}\left(A^{*}\left(v^{n^{\prime}(\tau)}\right)\right) \cdot n^{\prime}(\tau)}{2 c}\left(\bar{u}_{k_{\min }}\left(v^{n^{\prime}(\tau)}\right)-\bar{u}\left(v^{n^{\prime}(\tau)}\right)\right)\right)}
\end{aligned}
$$

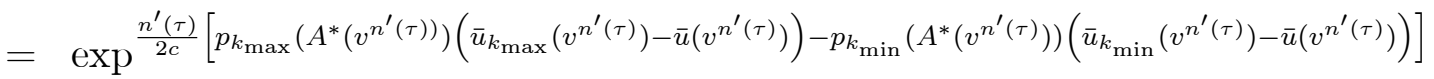

which, given Expression (8), is strictly greater than

$$
\begin{aligned}
& e^{\frac{n^{\prime}(\tau)}{2 c}\left[\delta\left(\bar{u}_{k \max }\left(v^{n^{\prime}(\tau)}\right)-\bar{u}\left(v^{n^{\prime}(\tau)}\right)\right)-\delta\left(\bar{u}\left(v^{n^{\prime}(\tau)}\right)-\bar{u}_{k_{\min }}\left(v^{n^{\prime}(\tau)}\right)\right)\right]} \\
= & e^{\frac{n^{\prime}(\tau)}{2 c} \delta\left(\bar{u}_{k_{\max }}\left(v^{n^{\prime}(\tau)}\right)-\bar{u}_{k_{\min }}\left(v^{n^{\prime}(\tau)}\right)\right)}
\end{aligned}
$$

for any $\tau>\hat{\tau}$. Further, since with probability one, $\lim _{\tau \longrightarrow \infty} \bar{u}_{k_{\max }}\left(v^{n^{\prime}(\tau)}\right)=u_{k_{\max }}$ and $\lim _{\tau \longrightarrow \infty} \bar{u}_{k_{\min }}\left(v^{n^{\prime}(\tau)}\right)=$ $u_{k_{\text {min }}}$, it follows that with probability one,

$$
\lim _{\tau \longrightarrow \infty} e^{\frac{n^{\prime}(\tau)}{2 c} \delta\left(\bar{u}_{k_{\max }}\left(v^{n^{\prime}(\tau)}\right)-\bar{u}_{k_{\min }}\left(v^{n^{\prime}(\tau)}\right)\right)}=\lim _{\tau \longrightarrow \infty} e^{\frac{n^{\prime}(\tau)}{2 c} \delta\left(u_{k_{\max }}-u_{k_{\min }}\right)} \longrightarrow \infty
$$


SO

$$
\lim _{\tau \longrightarrow \infty} \frac{p_{k_{\max }}\left(A^{*}\left(v^{n^{\prime}(\tau)}\right)\right)}{p_{k_{\min }}\left(A^{*}\left(v^{n^{\prime}(\tau)}\right)\right)} \longrightarrow \infty,
$$

which contradicts Expression (9).

Hence, there do not exist $\varepsilon \in \mathbb{R}_{++}$and a strictly increasing $n^{\prime}: \mathbb{N} \longrightarrow \mathbb{N}$ such that, for each $\tau \in \mathbb{N}$, there is a set of alternatives $K(\tau) \subset\{1,2, \ldots, m\}$ with $|K(\tau)|>1$, such that $p_{k}\left(A^{*}\left(v^{n^{\prime}(\tau)}\right)\right)>\varepsilon \Longleftrightarrow k \in K(\tau)$.

Equivalently, there exists $n(\varepsilon) \in \mathbb{N}$ such that for any $n>n(\varepsilon), \mid k \in X: p_{k}\left(A^{*}\left(v^{n}\right)\right)>$ $\varepsilon \mid=1$.

Lemma 3 establishes that one alternative wins with probability arbitrarily close to one if the size $n$ of the set of participating agents is sufficiently large. It does not rule out that this alternative, labeled $k\left(n, a^{*}(v)\right)$, could vary with $n$. However, since the set of alternatives is finite, we can identify a subsequence of sizes $\{n(t)\}_{t=1}^{\infty}$ such that along this subsequence, the alternative that wins with probability converging to one is always the same. We refer to this alternative as the "likely winner" and the label it as $w\left(a^{*}(v)\right){ }^{11}$

Corollary 2 For any $v \in V$, and for any sequence of equilibria $a^{*}(v) \in \prod_{n=2}^{\infty}[-1,1]^{m \times n}$, there exist an alternative $w\left(a^{*}(v)\right) \in X$ and a strictly increasing $n: \mathbb{N} \longrightarrow \mathbb{N}$ such that

$$
\lim _{t \longrightarrow \infty} p_{w\left(a^{*}(v)\right)}\left(A^{*}\left(v^{n(t)}\right)\right)=1
$$

Then $\left.\lim _{t \rightarrow \infty} p_{k}\left(A^{*}\left(v^{n(t)}\right)\right)\right)=0$ for any $k \in X \backslash\left\{w\left(a^{*}(v)\right)\right\}$. For the subsequent results, from Lemma 4 to Lemma 7, we work along the subsequence of sizes $\{n(t)\}_{t=1}^{\infty}$, and hence of games $\left\{\Gamma\left(v^{n(t)}\right)\right\}_{t=1}^{\infty}$. We show that along this subsequence, alternatives that are better than the likely winner receive positive net votes, and alternatives that are worse than the likely winner receive negative net votes. Recall that alternatives are ordered from best to worst: $u_{1}>u_{2}>\ldots>u_{m}$.

Lemma 4 For any $v \in V$, and for any sequence of equilibria $a^{*}(v) \in \prod_{t=1}^{\infty}[-1,1]^{m \times n(t)}$, there exists $\bar{t} \in \mathbb{N}$ such that for any $t>\bar{t}$,

$$
\left.A_{k}^{*}\left(v^{n(t)}\right)\right)>0 \text { if } k<w\left(a^{*}(v)\right) \text { and } A_{k}^{*}\left(v^{n(t)}\right)<0 \text { if } k>w\left(a^{*}(v)\right) .
$$

\footnotetext{
${ }^{11}$ While in its construction it appears possible that the identity of the likely winner alternative depends on the subsequence we choose, we will later prove that this alternative is the same, for any chosen sequence, hence the label it $w\left(a^{*}(v)\right)$ without reference to the chosen subsequence, without ambiguity.
} 
Proof. Recall from Expression (11) that for any $k \in X$,

$$
A_{k}^{*}\left(v^{n(t)}\right)=\frac{p_{k}\left(A^{*}\left(v^{n(t)}\right)\right) n(t)}{2 c} \sum_{h \in X}\left(\bar{u}_{k}\left(v^{n(t)}\right)-\bar{u}_{h}\left(v^{n(t)}\right)\right) p_{h}\left(A^{*}\left(v^{n(t)}\right)\right)
$$

and since by Corollary 2, $\lim _{t \longrightarrow \infty} p_{k}\left(A^{*}\left(v^{n(t)}\right)\right)=0$ for any $k \in X \backslash\left\{w\left(a^{*}(v)\right)\right\}$,

$\lim _{t \longrightarrow \infty} \sum_{h \in X}\left(\bar{u}_{k}\left(v^{n(t)}\right)-\bar{u}_{h}\left(v^{n(t)}\right)\right) p_{h}\left(A^{*}\left(v^{n(t)}\right)\right)=\lim _{t \longrightarrow \infty}\left(\bar{u}_{k}\left(v^{n(t)}\right)-\bar{u}_{w\left(a^{*}(v)\right)}\left(v^{n(t)}\right)\right) p_{w\left(a^{*}(v)\right)}\left(A^{*}\left(v^{n(t)}\right)\right)$

So

$$
\lim _{t \longrightarrow \infty} A_{k}^{*}\left(v^{n(t)}\right)>0 \Leftrightarrow \bar{u}_{k}\left(v^{n(t)}\right)-\bar{u}_{w\left(a^{*}(v)\right)}>0 \Longleftrightarrow k<w\left(a^{*}(v)\right) .
$$

Lemma 5 For any $v \in V$, and for any sequence of equilibria $a^{*}(v) \in \prod_{t=1}^{\infty}[-1,1]^{m \times n(t)}$, if the likely winner $w\left(a^{*}(v)\right)$ is not the best alternative (if $\left.w\left(a^{*}(v)\right) \neq 1\right)$, then $\lim _{t \rightarrow \infty} A_{w\left(a^{*}(v)\right)}^{*}\left(v^{n(t)}\right)=$ $\infty$.

Proof. If $w\left(a^{*}(v)\right)>1$, then by Lemma 4, there exists $\bar{t} \in \mathbb{N}$ such that for any $t>\bar{t}$, $A_{1}^{*}\left(v^{n(t)}\right)>0$. By Corollary 2, $\lim _{t \longrightarrow \infty} p_{w\left(a^{*}(v)\right)}\left(A^{*}\left(v^{n(t)}\right)\right)=1$, which implies that there exists $t^{\prime} \in \mathbb{N}$ such that $A_{w\left(a^{*}(v)\right)}^{*}\left(v^{n(t)}\right)>A_{k}^{*}\left(v^{n(t)}\right)$ for any $t>\tau^{\prime}$ and for any $k \in X \backslash\left\{w\left(a^{*}(v)\right)\right\}$, and in particular, $A_{w\left(a^{*}(v)\right)}^{*}\left(v^{n(t(\tau)}\right)>A_{1}^{*}\left(v^{n(t(\tau)}\right)$ for any $t>\tau^{\prime}$. Hence, for any $t>\max \left\{\bar{t}, t^{\prime}\right\}$, $A_{w\left(a^{*}(v)\right)}^{*}\left(v^{n(t)}\right)>A_{1}^{*}\left(v^{n(t)}\right)>0$ so $A_{w\left(a^{*}(v)\right)}^{*}\left(v^{n(t)}\right)>0$.

Assume (absurd) that there exist $\kappa \in \mathbb{R}_{++}$and a strictly increasing $t: \mathbb{N} \longrightarrow \mathbb{N}$ such that $A_{w\left(a^{*}(v)\right)}^{*}\left(v^{n(t(\tau)}\right) \leq \kappa$ for any $\tau \in \mathbb{N}$. Then $e^{A_{w\left(a^{*}(v)\right)}^{*}\left(v^{n(t(\tau)}\right)} \leq e^{\kappa}$. From Lemma 4, for any $t>\max \left\{\bar{t}, t^{\prime}\right\}$ and for any $k \in\left\{1, \ldots, w\left(a^{*}(v)\right)-1\right\}, A_{k}^{*}(n(t))>0$; and hence since $w\left(a^{*}(v)\right)>1$, it also follows that $\sum_{h=1}^{w\left(a^{*}(v)\right)} e^{A_{h}^{*}\left(v^{n(t)}\right)} \geq 1$ for any $t>\max \left\{\bar{t}, t^{\prime}\right\}$, so

$$
p_{w\left(a^{*}(v)\right)}\left(A^{*}\left(v^{n(t(\tau))}\right)\right) \leq \frac{e^{\kappa}}{e^{\kappa}+1} \text { for any } \tau \in \mathbb{N} \text { s.t. } t(\tau)>\max \left\{\bar{t}, t^{\prime}\right\}
$$

which contradicts Corollary 2.

Hence, there does not exist $\kappa \in \mathbb{R}_{++}$and a strictly increasing $t: \mathbb{N} \longrightarrow \mathbb{N}$ such that $A_{w\left(a^{*}(v)\right)}^{*}\left(v^{n(t(\tau))}\right) \leq \kappa$ for any $\tau \in \mathbb{N}$; or, equivalently, for any $\kappa \in \mathbb{R}_{++}, \exists t(\kappa) \in \mathbb{N}$ such that $A_{w\left(a^{*}(v)\right)}^{*}\left(v^{n(t)}\right)>\kappa$ for any $t>t(\kappa)$, so $\lim _{t \longrightarrow \infty} A_{w\left(a^{*}(v)\right)}^{*}\left(v^{n(t)}\right)=\infty$, as desired. 
Lemma 6 For any $v \in V$, and for any sequence of equilibria $a^{*}(v) \in \prod_{t=1}^{\infty}[-1,1]^{m \times n(t)}$, If $w\left(a^{*}(v)\right)>1$, there exists $k>w\left(a^{*}(v)\right)$ such that $\lim _{t \rightarrow \infty} A_{k}^{*}\left(v^{n(t)}\right)=-\infty$.

Proof. Since $v \in V$, there exists $\bar{t} \in \mathbb{N}$ such that $\bar{u}_{w\left(a^{*}(v)\right)}\left(v^{n(t)}\right)-\bar{u}_{k}\left(v^{n(t)}\right)<0$ for any $t>\bar{t}$, for any $k \in\left\{1, \ldots, w\left(a^{*}(v)\right)-1\right\}$, and thus if $w\left(a^{*}(v)\right)=m$ (the worst alternative), then $\bar{u}_{w\left(a^{*}(v)\right)}\left(v^{n(t)}\right)-\bar{u}_{k}\left(v^{n(t)}\right)<0$ for any $t>\bar{t}$, and for any $k \notin w\left(a^{*}(v)\right)$. From Expression (11), for any $t \in \mathbb{N}$,

$$
A_{w\left(a^{*}(v)\right)}^{*}\left(v^{n(t)}\right)=\frac{p_{w\left(a^{*}(v)\right)}\left(A^{*}\left(v^{n(t)}\right)\right) n(t)}{2 c} \sum_{k \in X}\left(\bar{u}_{w\left(a^{*}(v)\right)}\left(v^{n(t)}\right)-\bar{u}_{k}\left(v^{n(t)}\right)\right) p_{k}\left(A^{*}\left(v^{n(t)}\right)\right)
$$

and thus if $w\left(a^{*}(v)\right)=m$, then $A_{w\left(a^{*}(v)\right)}^{*}\left(v^{n(t)}\right)<0$ for any $t>\bar{t}$, for any $k \in\left\{1, \ldots, w\left(a^{*}(v)\right)-\right.$ $1\}$, which contradicts Lemma 5. Hence, $w\left(a^{*}(v)\right) \in\{1,2, \ldots, m-1\}$.

From Expression (11), for any $k>w\left(a^{*}(v)\right)$, and for any $t \in \mathbb{N}$,

$$
A_{k}^{*}\left({ }^{n(t)}\right)=\frac{p_{k}\left(A^{*}\left(v^{n(t)}\right)\right) n(t)}{2 c} \sum_{h \in X}\left(\bar{u}_{k}\left(v^{n(t)}\right)-\bar{u}_{h}\left(v^{n(t)}\right)\right) p_{h}\left(A^{*}\left(v^{n(t)}\right)\right),
$$

and since (by Corollary 2), $\lim _{t \rightarrow \infty} p_{h}\left(A^{*}(n(t))\right)=0$ for any $k \neq w\left(a^{*}(v)\right)$, it follows that for any $\varepsilon \in \mathbb{R}_{++}$, there exists $t(\varepsilon) \in \mathbb{N}$ such that for any $t>t(\varepsilon)$,

$$
A_{k}^{*}\left(v^{n(t)}\right)<\frac{p_{k}\left(A^{*}\left(v^{n(t)}\right)\right) n(t)}{2 c}\left(\bar{u}_{k}\left(v^{n(t)}\right)-\bar{u}_{w\left(a^{*}(v)\right)}\left(v^{n(t)}\right)+\varepsilon\right) .
$$

Summing up over all $k \in\left\{w\left(a^{*}(v)\right)+1, \ldots, m\right\}$,

$$
\sum_{k=w\left(a^{*}(v)\right)+1}^{m} A_{k}^{*}\left(v^{n(t)}\right)<\frac{n(t)}{2 c} \sum_{k=w\left(a^{*}(v)\right)+1}^{m} p_{k}\left(A^{*}\left(v^{n(t)}\right)\left(\bar{u}_{k}\left(v^{n(t)}\right)-\bar{u}_{w\left(a^{*}(v)\right)}\left(v^{n(t)}\right)+\varepsilon\right) .\right.
$$

Dividing both sides by

$$
-\frac{n(t)}{2 c} \sum_{k=w\left(a^{*}(v)\right)+1}^{m} p_{k}\left(A^{*}\left(v^{n(t)}\right)\left(\bar{u}_{k}\left(v^{n(t)}\right)-\bar{u}_{w\left(a^{*}(v)\right)}\left(v^{n(t)}\right)\right)\right.
$$


we obtain

$$
\begin{gathered}
-\frac{\sum_{k=w\left(a^{*}(v)\right)+1}^{m} A_{k}^{*}\left(v^{n(t)}\right)}{\frac{n(t)}{2 c} \sum_{k=w\left(a^{*}(v)\right)+1}^{m} p_{k}\left(A^{*}\left(v^{n(t)}\right)\left(\bar{u}_{k}\left(v^{n(t)}\right)-\bar{u}_{w\left(a^{*}(v)\right)}\left(v^{n(t)}\right)\right)\right.} \\
<-\frac{\sum_{k=w\left(a^{*}(v)\right)+1}^{m} p_{k}\left(A^{*}\left(v^{n(t)}\right)\left(\bar{u}_{k}\left(v^{n(t)}\right)-\bar{u}_{w\left(a^{*}(v)\right)}\left(v^{n(t)}\right)+\varepsilon\right)\right.}{\sum_{k=w\left(a^{*}(v)\right)+1}^{m} p_{k}\left(A^{*}\left(v^{n(t)}\right)\left(\bar{u}_{k}\left(v^{n(t)}\right)-\bar{u}_{w\left(a^{*}(v)\right)}\left(v^{n(t)}\right)\right)\right.} . \\
=-\frac{\sum_{k=w\left(a^{*}(v)\right)+1}^{m} \frac{p_{k}\left(A^{*}\left(v^{n(t)}\right)\right.}{\sum_{h=w\left(a^{*}(v)\right)+1}^{m} p_{h}\left(A^{*}\left(v^{n(t)}\right)\right)}\left(\bar{u}_{k}\left(v^{n(t)}\right)-\bar{u}_{w\left(a^{*}(v)\right)}\left(v^{n(t)}\right)+\varepsilon\right)}{\frac{p_{k}\left(A^{*}\left(v^{n(t)}\right)\right.}{\sum_{k=w\left(a^{*}(v)\right)+1}^{m} p_{h}\left(A^{*}\left(v^{n(t)}\right)\right)}\left(\bar{u}_{k}\left(v^{n(t)}\right)-\bar{u}_{w\left(a^{*}(v)\right)}\left(v^{n(t)}\right)\right)} .
\end{gathered}
$$

Note that dividing the numerator and denominator of the right hand side by $\sum_{h=w\left(a^{*}(v)\right)+1}^{m} p_{h}\left(A^{*}\left(v^{n(t)}\right)\right)$, the right hand side is equal to

$$
-1+\frac{\varepsilon}{\sum_{k=w\left(a^{*}(v)\right)+1}^{m}\left(\frac{p_{k}\left(A^{*}\left(v^{n(t)}\right)\right.}{\sum_{h=w\left(a^{*}(v)\right)+1}^{m} p_{h}\left(A^{*}\left(v^{n(t)}\right)\right)}\left(\bar{u}_{w\left(a^{*}(v)\right)}\left(v^{n(t)}\right)-\bar{u}_{k}\left(v^{n(t)}\right)\right)\right)} .
$$

Note that

$$
\sum_{k=w\left(a^{*}(v)\right)+1}^{m}\left(\frac{p_{k}\left(A^{*}\left(v^{n(t)}\right)\right.}{\sum_{h=w\left(a^{*}(v)\right)+1}^{m} p_{h}\left(A^{*}\left(v^{n(t)}\right)\right)}\left(\bar{u}_{w\left(a^{*}(v)\right)}\left(v^{n(t)}\right)-\bar{u}_{k}\left(v^{n(t)}\right)\right)\right)
$$

is a weighted average of $\left(\bar{u}_{w\left(a^{*}(v)\right)}\left(v^{n(t)}\right)-\bar{u}_{k}\left(v^{n(t)}\right)\right)$ across $k \in\left\{w\left(a^{*}(v)\right)+1, \ldots, m\right\}$. Since $v \in V$, there exists $\tilde{t}(v) \in \mathbb{N}$ such that

$$
\bar{u}_{k}\left(v^{n(t)}\right) \in\left(u_{k}-\frac{\min _{k \in\{1, \ldots, m-1\}}\left\{u_{k}-u_{k+1}\right\}}{3}, u_{k}+\frac{\min _{k \in\{1, \ldots, m-1\}}\left\{u_{k}-u_{k+1}\right\}}{3}\right)
$$

for any $t>\tilde{t}(v)$ and for each alternative $k$. Then, for any $t>\tilde{t}(v)$, and for any alternative 
$k \in\left\{w\left(a^{*}(v)\right)+1, \ldots, m\right\}$,

$$
\bar{u}_{w\left(a^{*}(v)\right)}\left(v^{n(t)}\right)-\bar{u}_{k}\left(v^{n(t)}\right)>\frac{\min _{k \in\{1, \ldots, m-1\}}\left\{u_{k}-u_{k+1}\right\}}{3} \in \mathbb{R}_{++} .
$$

Hence the denominator of Expression (15) is greater than $\frac{\min _{k \in\{1, \ldots, m-1\}}\left\{u_{k}-u_{k+1}\right\}}{3}$, so it is strictly

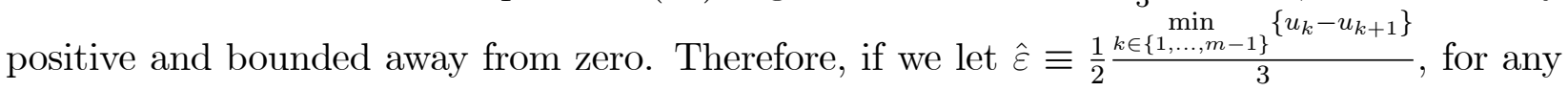
$t>\tilde{t}(v)$, and for $\varepsilon=\hat{\varepsilon}$, we find that $\hat{\varepsilon}<\frac{1}{2}$, and hence that Expression (14) is strictly less than $-1+\frac{1}{2}$. Since for any $\varepsilon \in \mathbb{R}_{++}$and any $t>t(\varepsilon)$ Expression (12) is strictly less than Expression (13), which is equal to Expression (14); and since for any $t>\tilde{t}(v)$ and for $\varepsilon=\hat{\varepsilon}$, Expression (14) is strictly less than $\frac{-1}{2}$, it follows that for any $t>\max \{\tilde{t}(v), t(\hat{\varepsilon})\}$, Expression (12) is strictly less than $-\frac{1}{2}$. That is, for any $t>\max \{\tilde{t}(v), t(\hat{\varepsilon})\}$,

$$
\frac{\sum_{k=w\left(a^{*}(v)\right)+1}^{m} A_{k}^{*}\left(v^{n(t)}\right)}{\frac{n(t)}{2 c} \sum_{k=w\left(a^{*}(v)\right)+1}^{m} p_{k}\left(A^{*}\left(v^{n(t)}\right)\left(\bar{u}_{k}\left(v^{n(t)}\right)-\bar{u}_{w\left(a^{*}(v)\right)}\left(v^{n(t)}\right)\right)\right.}<-\frac{1}{2},
$$

which implies that for any $t>\max \{\tilde{t}(v), t(\hat{\varepsilon})\}$,

$$
-2 \sum_{k=w\left(a^{*}(v)\right)+1}^{m} A_{k}^{*}\left(v^{n(t)}\right)>\frac{n(t)}{2 c} \sum_{k=w\left(a^{*}(v)\right)+1}^{m} p_{k}\left(A^{*}\left(v^{n(t)}\right)\left(\bar{u}_{w\left(a^{*}(v)\right)}\left(v^{n(t)}\right)-\bar{u}_{k}\left(v^{n(t)}\right)\right) .\right.
$$

Rearranging terms in Expression (11), and substituting into the right hand side of (16), this right hand side is equal to

$$
\frac{A_{w\left(a^{*}(v)\right)}^{*}\left(v^{n(t)}\right)}{p_{w\left(a^{*}(v)\right)}\left(A^{*}\left(v^{n(t)}\right)\right)}-\frac{n(t)}{2 c} \sum_{k=1}^{w\left(a^{*}(v)\right)-1} p_{k}\left(A^{*}\left(v^{n(t)}\right)\right)\left(\bar{u}_{w\left(a^{*}(v)\right)}\left(v^{n(t)}\right)-\bar{u}_{k}\left(v^{n(t)}\right)\right) .
$$

The first term of Expression (17) diverges to infinity in $t$ (the numerator diverges to infinity by Lemma 5 and the denominator is a probability that converges to one by Corollary 2) and the second term being subtracted is negative for sufficiently large $t$, because $v \in V$ implies that there exists $\bar{t} \in \mathbb{N}$ such that $\bar{u}_{w\left(a^{*}(v)\right)}\left(v^{n(t)}\right)<\bar{u}_{k}\left(v^{n(t)}\right)$ for any $t>\bar{t}$, for any $k<w\left(a^{*}(v)\right)$. Hence, Expression (17) diverges to infinity. Hence, $\sum_{k=w\left(a^{*}(v)\right)+1}^{m} A_{k}^{*}\left(v^{n(t)}\right)$ diverges to $-\infty$, which implies that there exists $k \in\left\{w\left(a^{*}(v)\right)+1, \ldots, m\right\}$ such that $\left.\lim _{t \longrightarrow \infty} A_{k}^{*}\left(v^{n(t)}\right)\right)=-\infty$.

Let $l \in\left\{w\left(a^{*}(v)\right)+1, \ldots, m\right\}$ be an alternative such that $\lim _{t \longrightarrow \infty} A_{l}^{*}\left(v^{n(t)}\right)=-\infty$. 
Corollary 3 For any $v \in V$, and for any sequence of equilibria $a^{*}(v) \in \prod_{t=1}^{\infty}[-1,1]^{m \times n(t)}$, if $w\left(a^{*}(v)\right)>1$, there exists a strictly increasing $t: \mathbb{N} \longrightarrow \mathbb{N}$ such that for any $\tau \in \mathbb{N}$, $A_{l}^{*}\left(v^{n(t(\tau+1))}\right)<A_{l}^{*}\left(v^{n(t(\tau))}\right)$.

That is, along subsequence $\{n(t(\tau))\}_{\tau=1}^{\infty}$, alternative $l$ receives monotonically decreasing aggregate net actions, diverging toward $-\infty$. We next show that the aggregate actions on all other alternatives worse than $w$ also diverge to $-\infty$ along this subsequence.

Lemma 7 For any $v \in V$, and for any sequence of equilibria $a^{*}(v) \in \prod_{\tau=1}^{\infty}[-1,1]^{m \times n(t(\tau))}$, and for any $k \in\left\{w\left(a^{*}(v)\right)+1, \ldots, m\right\}, \lim _{\tau \longrightarrow \infty} A_{k}^{*}\left(v^{n(t(\tau))}\right)=-\infty$.

Proof. Proof by contradiction. Assume (absurd) that there exists $k \in\left\{w\left(a^{*}(v)\right)+1, \ldots, m\right\}$, $\kappa \in \mathbb{R}$ and a strictly increasing $\tau: \mathbb{N} \longrightarrow \mathbb{N}$ such that $A_{k}^{*}\left(v^{n(t(\tau(\gamma)))}\right) \geq \kappa$ for any $\gamma \in \mathbb{N}$. Then

$$
\begin{aligned}
\frac{A_{l}^{*}\left(v^{n(t(\tau(\gamma)))}\right)}{A_{k}^{*}\left(v^{n(t(\tau(\gamma)))}\right)}=\frac{p_{l}\left(A^{*}\left(v^{n(t(\tau(\gamma)))}\right)\right) \sum_{h \in X}\left(\bar{u}_{l}\left(v^{n(t(\tau(\gamma)))}\right)-\bar{u}_{h}\left(v^{n(t(\tau(\gamma)))}\right)\right) p_{h}\left(A^{*}\left(v^{n(t(\tau(\gamma)))}\right)\right)}{p_{k}\left(A^{*}\left(v^{n(t(\tau(\gamma)))}\right)\right) \sum_{h \in X}\left(\bar{u}_{k}\left(v^{n(t(\tau(\gamma)))}\right)-\bar{u}_{h}\left(v^{n(t(\tau(\gamma)))}\right)\right) p_{h}\left(A^{*}\left(v^{n(t(\tau(\gamma)))}\right)\right)} \\
=\frac{p_{l}\left(A^{*}\left(v^{n(t(\tau(\gamma)))}\right)\right)\left(\bar{u}_{l}\left(v^{n(t(\tau(\gamma)))}\right)-\sum_{h \in X} \bar{u}_{h}\left(v^{n(t(\tau(\gamma)))}\right) p_{h}\left(A^{*}\left(v^{n(t(\tau(\gamma)))}\right)\right)\right)}{p_{k}\left(A^{*}\left(v^{n(t(\tau(\gamma)))}\right)\right)\left(\bar{u}_{k}\left(v^{n(t(\tau(\gamma)))}\right)-\sum_{h \in X} \bar{u}_{h}\left(v^{n(t(\tau(\gamma)))}\right) p_{h}\left(A^{*}\left(v^{n(t(\tau(\gamma)))}\right)\right)\right)},
\end{aligned}
$$

which is equal to

$$
\left(\exp \left(A_{l}^{\left.*\left(v^{n(t(\tau(\gamma)))}\right)-A_{k}^{*}\left(v^{n(t(\tau(\gamma)))}\right)\right)}\right) \frac{\bar{u}_{l}\left(v^{n(t(\tau(\gamma)))}\right)-\sum_{h \in X} \bar{u}_{h}\left(v^{n(t(\tau(\gamma)))}\right) p_{h}\left(A^{*}\left(v^{n(t(\tau(\gamma)))}\right)\right)}{\bar{u}_{k}\left(v^{n(t(\tau(\gamma)))}\right)-\sum_{h \in X} \bar{u}_{h}\left(v^{n(t(\tau(\gamma)))}\right) p_{h}\left(A^{*}\left(v^{n(t(\tau(\gamma)))}\right)\right)} .\right.
$$

By Corollary 2,

$$
\lim _{\gamma \longrightarrow \infty} \sum_{h \in X} \bar{u}_{h}\left(v^{n(t(\tau(\gamma)))}\right) p_{h}\left(A^{*}\left(v^{n(t(\tau(\gamma)))}\right)=\lim _{\gamma \longrightarrow \infty} \bar{u}_{w\left(a^{*}(v)\right)}\left(v^{n(t(\tau(\gamma)))}\right),\right.
$$

and since $v \in V, \lim _{\gamma \longrightarrow \infty} \bar{u}_{h}\left(v^{n(t(\tau(\gamma)))}\right)=u_{h}$ for $h \in\left\{k, l, w\left(a^{*}(v)\right\}\right.$,

$$
\lim _{\gamma \longrightarrow \infty} \frac{\bar{u}_{l}\left(v^{n(t(\tau(\gamma)))}\right)-\sum_{h \in X} \bar{u}_{h}\left(v^{n(t(\tau(\gamma)))}\right) p_{h}\left(A^{*}\left(v^{n(t(\tau(\gamma)))}\right)\right)}{\bar{u}_{k}\left(v^{n(t(\tau(\gamma)))}\right)-\sum_{h \in X} \bar{u}_{h}\left(v^{n(t(\tau(\gamma)))}\right) p_{h}\left(A^{*}\left(v^{n(t(\tau(\gamma)))}\right)\right)}=\frac{u_{w\left(a^{*}(v)\right)}-u_{l}}{u_{w\left(a^{*}(v)\right)}-u_{k}} \in \mathbb{R},
$$


so in particular, the ratio does not diverge and remains bounded. Further, since $\lim _{\gamma \longrightarrow \infty} A_{l}^{*}\left(v^{n(t(\tau(\gamma)))}\right)=$ $-\infty$ and $A_{k}^{*}\left(v^{n(t(\tau(\gamma)))}\right) \geq \kappa$ for any $\gamma \in \mathbb{N}$, it follows $\lim _{\gamma \longrightarrow \infty}\left(A_{l}^{*}\left(v^{n(t(\tau(\gamma)))}\right)-A_{k}^{*}\left(v^{n(t(\tau(\gamma)))}\right)=\right.$ $-\infty$ and thus

$$
\lim _{\gamma \longrightarrow \infty} e^{\left(A_{l}^{*}\left(v^{n(t(\tau(\gamma)))}\right)-A_{k}^{*}\left(v^{n(t(\tau(\gamma)))}\right)\right)}=0 .
$$

Hence, taking the limits on both sides of Expression (18), and substituting in Expression (19) and Expression (20) in the right hand side,

$$
\begin{aligned}
\lim _{\gamma \longrightarrow \infty} \frac{A_{l}^{*}\left(v^{n(t(\tau(\gamma)))}\right)}{A_{k}^{*}\left(v^{n(t(\tau(\gamma)))}\right)}= & \left(\lim _{\gamma \longrightarrow \infty}\left(\exp ^{\left(A_{l}^{*}\left(v^{n(t(\tau(\gamma)))}\right)-A_{k}^{*}\left(v^{n(t(\tau(\gamma)))}\right)\right)}\right)\right) \\
& \left(\frac{\bar{u}_{l}\left(v^{n(t(\tau(\gamma)))}\right)-\sum_{h \in X} \bar{u}_{h}\left(v^{n(t(\tau(\gamma)))}\right) p_{h}\left(A^{*}\left(v^{n(t(\tau(\gamma)))}\right)\right)}{\bar{u}_{k}\left(v^{n(t(\tau(\gamma)))}\right)-\sum_{h \in X} \bar{u}_{h}\left(v^{n(t(\tau(\gamma)))}\right) p_{h}\left(A^{*}\left(v^{n(t(\tau(\gamma)))}\right)\right)}\right) \\
= & 0 .
\end{aligned}
$$

However, $\lim _{\gamma \longrightarrow \infty} A_{l}^{*}\left(v^{n(t(\tau(\gamma)))}\right)=-\infty$ and $A_{k}^{*}\left(v^{n(t(\tau(\gamma)))}\right) \geq \kappa$ for any $\gamma \in \mathbb{N}$ (by assumption), implies that

$$
\lim _{\gamma \longrightarrow \infty}\left|\frac{A_{l}^{*}\left(v^{n(t(\tau(\gamma)))}\right)}{A_{k}^{*}\left(v^{n(t(\tau(\gamma)))}\right)}\right|=\infty,
$$

a contradiction, so the initial assumption is false. Thus, for any $k>w\left(a^{*}(v)\right), \lim _{\tau \longrightarrow \infty} A_{k}^{*}(n(t(\tau)))=$ $-\infty$.

Corollary 4 For any $v \in V$, and for any sequence of equilibria $a^{*}(v) \in \prod_{t=1}^{\infty}[-1,1]^{m \times n(t(\tau))}$, if $w\left(a^{*}(v)\right)>1$, there exists a strictly increasing $\tau: \mathbb{N} \longrightarrow \mathbb{N}$ such that for any $\gamma \in \mathbb{N}$, and for any $k \in\left\{w\left(a^{*}(v)\right)+1, \ldots, m\right\}, A_{k}^{*}\left(v^{n(t(\tau(\gamma+1)))}\right)<A_{k}^{*}\left(v^{n(t(\tau(\gamma)))}\right)$.

That is, we have identified a subsequence $\{n(t(\tau(\gamma)))\}_{\gamma=1}^{\infty}$ such that the aggregate actions on all alternatives worse than $w\left(a^{*}(v)\right)$ are strictly decreasing toward minus infinity along the subsequence.

Proposition 1 The quadratic voting mechanism is asymptotically optimal.

Proof. Recall from Expression (11) that for any $v \in V$, for any sequence of equilibria $a^{*}(v) \in \prod_{n=2}^{\infty}[-1,1]^{m \times n}$, and for any $n \in \mathbb{N} \backslash\{1\}$,

$$
A_{w\left(a^{*}(v)\right)}^{*}\left(v^{n}\right)=\frac{p_{w\left(a^{*}(v)\right)}\left(A^{*}\left(v^{n}\right)\right) n}{2 c} \sum_{k \in X}\left(\bar{u}_{w\left(a^{*}(v)\right)}\left(v^{n}\right)-\bar{u}_{k}\left(v^{n}\right)\right) p_{k}\left(A^{*}\left(v^{n}\right)\right)
$$


so

$$
\frac{A_{w\left(a^{*}(v)\right)}^{*}\left(v^{n}\right) 2 c}{p_{w\left(a^{*}(v)\right)}\left(A^{*}\left(v^{n}\right)\right) n}=\sum_{k \in X}\left(\bar{u}_{w\left(a^{*}(v)\right)}\left(v^{n}\right)-\bar{u}_{k}\left(v^{n}\right)\right) \frac{e^{A_{k}^{*}\left(v^{n}\right)}}{\sum_{j \in X} e^{A_{j}^{*}\left(v^{n}\right)}}
$$

and

$$
\frac{A_{w\left(a^{*}(v)\right)}^{*}\left(v^{n}\right) 2 c}{p_{w\left(a^{*}(v)\right)}\left(A^{*}\left(v^{n}\right)\right) n} \sum_{j \in X} e^{A_{j}^{*}\left(v^{n}\right)}=\sum_{k \in X}\left(\bar{u}_{w\left(a^{*}(v)\right)}\left(v^{n}\right)-\bar{u}_{k}\left(v^{n}\right)\right) e^{A_{k}^{*}\left(v^{n}\right)} .
$$

which, decomposing the right-hand side, is equal to

$$
\sum_{k=1}^{w\left(a^{*}(v)\right)-1}\left(\bar{u}_{w\left(a^{*}(v)\right)}\left(v^{n}\right)-\bar{u}_{k}\left(v^{n}\right)\right) e^{A_{k}^{*}\left(v^{n}\right)}+\sum_{k=w\left(a^{*}(v)\right)+1}^{m}\left(\bar{u}_{w\left(a^{*}(v)\right)}\left(v^{n}\right)-\bar{u}_{k}\left(v^{n}\right)\right) e^{A_{k}^{*}\left(v^{n}\right)} .
$$

Since $\lim _{\gamma \longrightarrow \infty} A_{k}^{*}(n(t(\tau(\gamma))))=-\infty$ (Lemma 7 and Corollary 4), it follows that $\lim _{\gamma \longrightarrow \infty} e^{A_{k}^{*}\left(v^{n(t(\tau(\gamma)))}\right)}=$ 0 so the second term of Expression (22) converges to zero along the subsequence $\{n(t(\tau(\gamma)))\}_{\gamma=1}^{\infty}$. Hence,

$$
\begin{aligned}
& \lim _{\gamma \longrightarrow \infty}\left(\begin{array}{c}
\sum_{k=1}^{w\left(a^{*}(v)\right)-1}\left(\bar{u}_{w\left(a^{*}(v)\right)}\left(v^{n(t(\tau(\gamma)))}\right)-\bar{u}_{k}\left(v^{n(t(\tau(\gamma)))}\right)\right) e^{A_{k}^{*}\left(v^{n(t(\tau(\gamma)))}\right)} \\
+\sum_{k=w\left(a^{*}(v)\right)+1}^{m}\left(\bar{u}_{w\left(a^{*}(v)\right)}\left(v^{n(t(\tau(\gamma)))}\right)-\bar{u}_{k}\left(v^{n(t(\tau(\gamma)))}\right)\right) e^{A_{k}^{*}\left(v^{n(t(\tau(\gamma)))}\right)}
\end{array}\right) \\
& =\lim _{\gamma \longrightarrow \infty}\left(\sum_{k=1}^{w\left(a^{*}(v)\right)-1}\left(\bar{u}_{w\left(a^{*}(v)\right)}\left(v^{n(t(\tau(\gamma)))}\right)-\bar{u}_{k}\left(v^{n(t(\tau(\gamma)))}\right)\right) e^{A_{k}^{*}\left(v^{n(t(\tau(\gamma)))}\right)}\right) \text {. }
\end{aligned}
$$

If $w\left(a^{*}(v)\right)>1$, then for any $k \in\left\{1, \ldots, w\left(a^{*}(v)\right)-1\right\}$, there exists $\bar{\gamma} \in \mathbb{N}$ such that for any $\gamma>\bar{\gamma}, \bar{u}_{w\left(a^{*}(v)\right)}\left(v^{n(t(\tau(\gamma)))}\right)<\bar{u}_{k}\left(v^{n(t(\tau(\gamma)))}\right)$ (because $v \in V$ ) and $A_{k}^{*}\left(v^{n(t(\tau(\gamma)))}\right)>0$ (by Lemma 4), which implies $e^{A_{k}^{*}\left(v^{n}(t(\tau(\gamma)))\right)}>1$. So for any $\gamma>\bar{\gamma}$,

$$
\begin{gathered}
\sum_{\substack{k=1 \\
w\left(a^{*}(v)\right)-1}}^{w\left(a^{*}(v)\right)-1}\left(\bar{u}_{w\left(a^{*}(v)\right)}\left(v^{n(t(\tau(\gamma)))}\right)-\bar{u}_{k}\left(v^{n(t(\tau(\gamma)))}\right)\right) e^{A_{k}^{*}\left(v^{n(t(\tau(\gamma)))}\right)} \\
<\sum_{k=1}\left(\bar{u}_{w\left(a^{*}(v)\right)}\left(v^{n(t(\tau(\gamma)))}\right)-\bar{u}_{k}\left(v^{n(t(\tau(\gamma)))}\right)\right)<\Theta
\end{gathered}
$$

for some $\Theta<0$.

Therefore, if $w\left(a^{*}(v)\right)>1$, Expression (23) is negative, and thus, from equalities (21), (22) and $(23), A_{w\left(a^{*}(v)\right)}^{*}\left(v^{n(t(\tau(\gamma)))}\right)<0$ for any $\gamma>\bar{\gamma}$, for any $v \in V$, and for any sequence of equilibria $a^{*}(v) \in \prod_{n=2}^{\infty}[-1,1]^{m \times n}$. But $A_{w\left(a^{*}(v)\right)}^{*}(n(t(\tau(\gamma))))<0$ together with $A_{k}^{*}(n(t(\tau(\gamma))))>0$ 
for $k<w\left(a^{*}(v)\right)$ contradicts the definition of $w\left(a^{*}(v)\right)$ as the likely winner: the likely winner must have the most votes. Thus, it follows that $w\left(a^{*}(v)\right)=1$, as desired. Recall that the likely winner $w\left(a^{*}(v)\right)$ was first defined with respect to a particular subsequence of society sizes $\{n(t)\}_{t=1}^{\infty}$ so that it remain constant within this sequence. The definition allowed that the likely winner be different for a different subsequence. Now we have established that for an arbitrary subsequence, the likely winner is alternative 1 . This applies to all subsequences, which establishes that the likely winner is in fact, $w\left(a^{*}(v)\right)=1$ for all subsequences, as desired.

We next note that actions, and hence transfers, converge to zero in the number of participants.

Proposition 2 For any $v \in V$, and for any sequence of equilibria $a^{*}(v)$, and for any $i \in \mathbb{N}$, $\lim _{n \longrightarrow \infty} a^{*, i}\left(v^{n}\right) \longrightarrow 0$, and thus agent $i^{\prime} s$ monetary contribution to the mechanism converges to zero.

Proof. This follows as a corollary from Lemma 3, which finds that for any $n>n(\varepsilon)$, there is one alternative $k\left(n, a^{*}(v)\right)$ such that $\lim _{n \longrightarrow \infty} p_{k\left(n, a^{*}(v)\right)}\left(A^{*}\left(v^{n}\right)\right) \longrightarrow 1$; and from Expression (10), which finds that for each $k \in X$ and each $i \in\{1, \ldots, n\}$,

$$
a_{k}^{*, i}\left(v^{n}\right)=\frac{p_{k}\left(A^{*}\left(v^{n}\right)\right)}{2 c} \sum_{h \in X \backslash\{k\}}\left(u_{k}^{i}-u_{h}^{i}\right) p_{h}\left(A^{*}\left(v^{n}\right)\right),
$$

hence, taking limits,

$$
\begin{aligned}
\lim _{n \longrightarrow \infty} a_{k}^{*, i}\left(v^{n}\right) & =\lim _{n \longrightarrow \infty} \frac{p_{k}\left(A^{*}\left(v^{n}\right)\right)}{2 c} \sum_{h \in X \backslash\{k\}}\left(u_{k}^{i}-u_{h}^{i}\right) p_{h}\left(A^{*}\left(v^{n}\right)\right), \\
& =0 .
\end{aligned}
$$

Finally, we prove that the equilibrium net aggregate actions asymptotically correspond to the full order of alternatives from best to worst.

Proposition 3 For any $v \in V$, and for any sequence of equilibria $a^{*}(v) \in \prod_{t=1}^{\infty}[-1,1]^{m \times n(t(\tau))}$, there exists $\hat{t} \in \mathbb{N}$ such that for any alternative $k \in\{1, \ldots, m-1\}$, and for any $t>\hat{t}$, $A_{k}^{*}\left(v^{n(t)}\right)>A_{k+1}^{*}\left(v^{n(t)}\right)$. 
Proof. From Expression (11), for any $k \in\{2, \ldots, m-1\}$, and for each $h \in\{k, k+1\}$,

$$
\begin{aligned}
A_{h}^{*}\left(v^{n(t)}\right) & =\frac{p_{h}\left(A^{*}\left(v^{n(t)}\right)\right) n(t)}{2 c} \sum_{l \in X}\left(\bar{u}_{h}\left(v^{n(t)}\right)-\bar{u}_{l}\left(v^{n(t)}\right) p_{l}\left(A^{*}\left(v^{n(t)}\right)\right),\right. \text { so } \\
\frac{2 c A_{h}^{*}\left(v^{n(t)}\right)}{p_{h}\left(A^{*}\left(v^{n(t)}\right)\right) n(t)} & =\sum_{l \in X}\left(\bar{u}_{h}\left(v^{n(t)}\right)-\bar{u}_{l}\left(v^{n(t)}\right) p_{l}\left(A^{*}\left(v^{n(t)}\right)\right) .\right.
\end{aligned}
$$

Since $\lim _{t \longrightarrow \infty} p_{l}\left(A^{*}\left(v^{n(t)}\right)\right)=0$ for any $l>1$, it follows that for each $h \in\{k, k+1\}$,

$$
\lim _{t \longrightarrow \infty} \frac{2 c A_{h}^{*}\left(v^{n(t)}\right)}{p_{h}\left(A^{*}\left(v^{n(t)}\right)\right) n(t)}=u_{h}-u_{1}
$$

and thus dividing $\lim _{t \longrightarrow \infty} \frac{2 c A_{h}^{*}\left(v^{n(t)}\right)}{p_{k}\left(A^{*}\left(v^{n(t)}\right)\right) n(t)}$ by $\lim _{t \longrightarrow \infty} \frac{2 c A_{h}^{*}\left(v^{n(t)}\right)}{p_{k+1}\left(A^{*}\left(v^{n(t)}\right)\right) n(t)}$ and rearranging terms, we obtain

$$
\begin{aligned}
\lim _{t \longrightarrow \infty} \frac{A_{k}^{*}\left(v^{n(t)}\right)}{A_{k+1}^{*}\left(v^{n(t)}\right)} \frac{p_{k+1}\left(A^{*}\left(v^{n(t)}\right)\right)}{p_{k}\left(A^{*}\left(v^{n(t)}\right)\right)} & =\frac{u_{k}-u_{1}}{u_{k+1}-u_{1}}, \text { and hence } \\
\lim _{t \longrightarrow \infty} \frac{A_{k}^{*}\left(v^{n(t)}\right)}{A_{k+1}^{*}\left(v^{n(t)}\right)} e^{\left(A_{k+1}^{*}\left(v^{n(t)}\right)-A_{k}^{*}\left(v^{n(t)}\right)\right)} & =\frac{u_{k}-u_{1}}{u_{k+1}-u_{1}}=\frac{u_{1}-u_{k}}{u_{1}-u_{k+1}} \in(0,1) .
\end{aligned}
$$

By Lemma 4, there exists $\bar{t} \in \mathbb{N}$ such that for any $t>\bar{t}, A_{k}^{*}\left(v^{n(t)}\right)<0$ and $A_{k+1}^{*}\left(v^{n(t)}\right)<0$. So for any $t>\bar{t}$,

$$
\begin{aligned}
A_{k+1}^{*}\left(v^{n(t)}\right) & \geq A_{k}^{*}\left(v^{n(t)}\right) \Longrightarrow \frac{A_{k}^{*}\left(v^{n(t)}\right)}{A_{k+1}^{*}\left(v^{n(t)}\right)} \geq 1 \Longrightarrow e^{\left(A_{k+1}^{*}\left(v^{n(t)}\right)-A_{k}^{*}\left(v^{n(t)}\right)\right)} \geq 1 \\
& \Longrightarrow \frac{A_{k}^{*}\left(v^{n(t)}\right)}{A_{k+1}^{*}\left(v^{n(t)}\right)} e^{\left(A_{k+1}^{*}\left(v^{n(t)}\right)-A_{k}^{*}\left(v^{n(t)}\right)\right)} \geq 1
\end{aligned}
$$

Taking the contrapositive, for any $t>\bar{t}$,

$$
\frac{A_{k}^{*}\left(v^{n(t)}\right)}{A_{k+1}^{*}\left(v^{n(t)}\right)} e^{\left(A_{k+1}^{*}\left(v^{n(t)}\right)-A_{k}^{*}\left(v^{n(t)}\right)\right)}<1 \Longrightarrow A_{k+1}^{*}\left(v^{n(t)}\right)<A_{k}^{*}\left(v^{n(t)}\right) .
$$

Since, by Expression (24),

$$
\lim _{t \longrightarrow \infty} \frac{A_{k}^{*}\left(v^{n(t)}\right)}{A_{k+1}^{*}\left(v^{n(t)}\right)} e^{\left(A_{k+1}^{*}\left(v^{n(t)}\right)-A_{k}^{*}\left(v^{n(t)}\right)\right)} \in(0,1),
$$

it follows that there is $\hat{t} \in \mathbb{N}$ such that for any $t>\hat{t}, A_{k+1}^{*}\left(v^{n(t)}\right)<A_{k}^{*}\left(v^{n(t)}\right)$, as desired. 


\section{References}

[1] Börgers, Tilmann. "Costly voting." American Economic Review 94(1): 57-66, 2004.

[2] Börgers, Tilman. 2015. An introduction to the theory of mechanism design. Oxford University Press.

[3] Börgers, Tilman, and Jiangtao Li. 2018. "Strategically simple mechanisms." Working paper.

[4] Brandt, Felix, Vincent Conitzer, Ulle Endriss, Jérôme Lang, and Ariel D. Procaccia, eds. 2016. Handbook of computational social choice. Cambridge University Press.

[5] Brânzei, Simina, Ioannis Caragiannis, Jamie Morgenstern, and Ariel D. Procaccia. 2013. "How bad is selfish voting?" Twenty-Seventh AAAI Conference on Artificial Intelligence.

[6] d'Aspremont, Claude, and Louis-André Gérard-Varet. 1979. "Incentives and incomplete information." Journal of Public Economics 11(1): 25-45.

[7] Casella, Alessandra. 2005. "Storable votes." Games and Economic Behavior 51(2): 391419.

[8] Conitzer, Vincent, Tuomas Sandholm, and Jérôme Lang. 2007. "When are elections with few candidates hard to manipulate?" Journal of the ACM 54(3): 14.

[9] Eguia, Jon, and Dimitrios Xefteris, "Implementation by vote-buying mechanisms." SSRN wp 3138614.

[10] Faliszewski, Piotr, and Ariel D. Procaccia. 2010. "AI's war on manipulation: Are we winning?" AI Magazine 31(4): 53-64.

[11] Gerard Debreu. 1952. "A social equilibrium existence theorem." Proceedings of the National Academy of Sciences 38: 886-893.

[12] Goeree, Jacob K., and Jingjing Zhang. 2017. "One man, one vote bid." Games and Economic Behavior 101: 151-171.

[13] Maskin, Eric, and Tomas Sjöström. 2002. "Implementation theory." Handbook of Social Choice and Welfare 1: 237-288.

[14] Jackson, Matthew O. 1992. "Implementation in undominated strategies: A look at bounded mechanisms." Review of Economic Studies 59(4): 757-775. 
[15] Jackson, Mathew O. 2000. "Mechanism theory." SSRN wp 2542983.

[16] Jackson, Matthew O. 2001. "A crash course in implementation theory." Social choice and welfare 18(4): $655-708$.

[17] Krishna, Vijay and John Morgan. 2015. "Majority rule and utilitarian welfare." American Economic Journal: Microeconomics 7(4): 339-375.

[18] Lalley, Steven P., and E. Glen Weyl. 2016. "Quadratic voting." SSRN wp 2003531.

[19] Lalley, Steven P., and E. Glen Weyl. 2018. "Quadratic voting: How mechanism design can radicalize democracy." AEA Papers and Proceedings 108: 33-37.

[20] Li, Shengwu. 2017. "Obviously strategy-proof mechanisms." American Economic Review 107(11): 3257-87.

[21] Maskin, Eric. 1999. "Nash equilibrium and welfare optimality." Review of Economic Studies 66(1): 23-38.

[22] Meir, Reshef. 2018. "Strategic Voting." Synthesis Lectures on Artificial Intelligence and Machine Learning 13(1): 1-167.

[23] Penrose, Lionel S. 1946. "The elementary statistics of majority voting." Journal of the Royal Statistical Society 109(1): 53-57.

[24] Simon, Carl, and Lawrence Blume. 1994. Mathematics for economists. Norton.

[25] Vickrey, William.1961. "Counterspeculation, auctions, and competitive sealed tenders." Journal of Finance. 16(1): 8-37.

[26] Clarke, Edward H. 1971. "Multipart pricing of public goods." Public Choice. 11(1): 17-33.

[27] Groves, Theodore. 1973. "Incentives in Teams." Econometrica 41(4): 617-631. 